Review

\title{
Moment-to-moment brain signal variability: A next frontier in human brain mapping?
}

\author{
Douglas D. Garrett ${ }^{\mathrm{a}, \mathrm{b}, *}$, Gregory R. Samanez-Larkin ${ }^{\mathrm{c}}$, Stuart W.S. MacDonald ${ }^{\mathrm{d}}$, Ulman Lindenberger ${ }^{\mathrm{a}, \mathrm{b}}$, \\ Anthony R. McIntosh e,f, Cheryl L. Grady e,f,g
}

${ }^{a}$ Max Planck Society-University College London Initiative: Computational Psychiatry and Aging Research (ICPAR)

b Center for Lifespan Psychology, Max Planck Institute for Human Development, Lentzeallee 94, 14195 Berlin, Germany

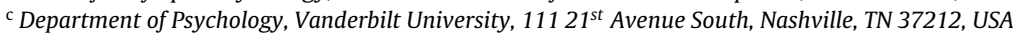

d Department of Psychology, University of Victoria, PO Box 3050 STN CSC, Victoria, BC V8W 3P5, Canada

e Rotman Research Institute, Baycrest, 3560 Bathurst Street, Toronto, Ontario M6A 2E1, Canada

${ }^{\mathrm{f}}$ Department of Psychology, University of Toronto, 100 St. George Street, Toronto, ON M5S 3G3, Canada

${ }^{g}$ Department of Psychiatry, University of Toronto, 250 College Street, Toronto, ON M5T 1R8, Canada

\section{A R T I C L E I N F O}

\section{Article history:}

Received 11 November 2012

Received in revised form 13 February 2013

Accepted 19 February 2013

\section{Keywords:}

Brain signal variability

Noise

Complexity

Dynamics

fMRI

EEG

MEG

\begin{abstract}
A B S T R A C T
Neuroscientists have long observed that brain activity is naturally variable from moment-to-moment, but neuroimaging research has largely ignored the potential importance of this phenomenon. An emerging research focus on within-person brain signal variability is providing novel insights, and offering highly predictive, complementary, and even orthogonal views of brain function in relation to human lifespan development, cognitive performance, and various clinical conditions. As a result, brain signal variability is evolving as a bona fide signal of interest, and should no longer be dismissed as meaningless noise when mapping the human brain.
\end{abstract}

(c) 2013 Elsevier Ltd. All rights reserved.

\section{Contents}

1. Brain activity is variable, and variability is often functional . . . . .

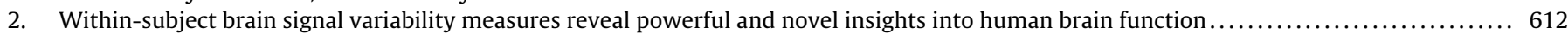

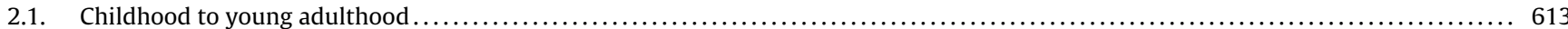

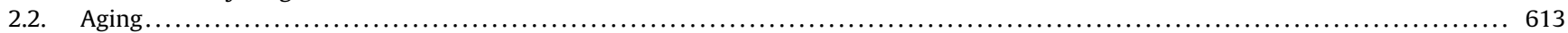

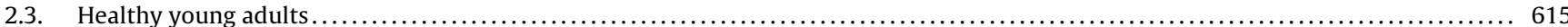

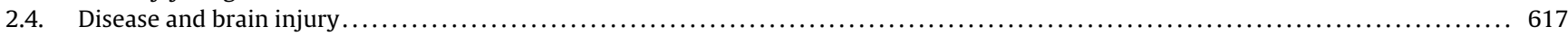

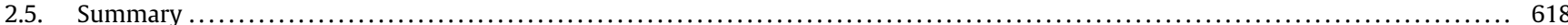

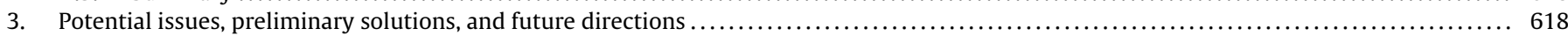

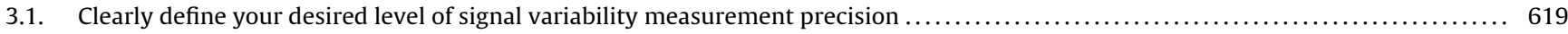

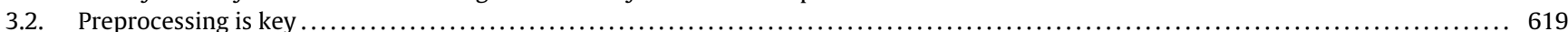

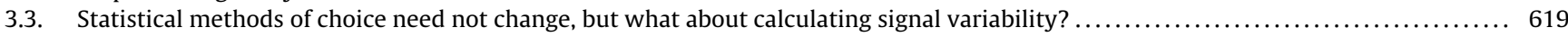

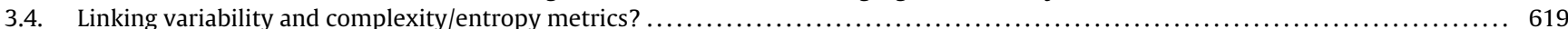

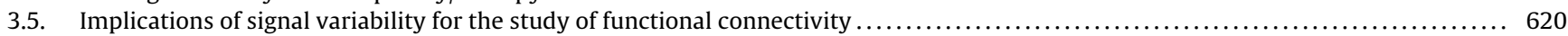

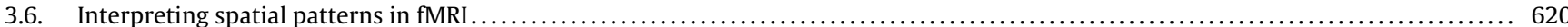

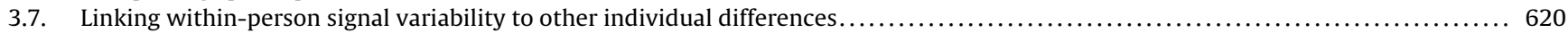

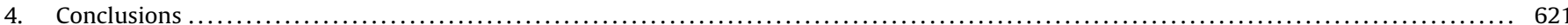

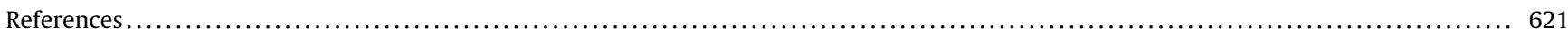

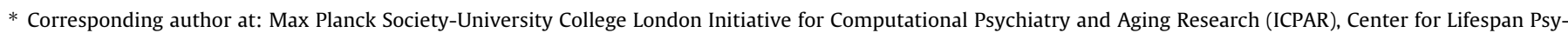
chology, Max Planck Institute for Human Development, Lentzeallee 94, 14195 Berlin, Germany. Tel.: +49 3082406216 ; fax: +49 308249939.

E-mail address: garrett@mpib-berlin.mpg.de (D.D. Garrett).
} 
Recent progress in cognitive neuroscience has been tremendous, largely due to the use of noninvasive forms of functional neuroimaging over the last several decades (primarily functional magnetic resonance imaging (fMRI), electroencephalography (EEG), and magnetoencephalography (MEG)). The examination of neuroimaging data is naturally complex, involving many voxels/electrodes/sensors and time points, at each of which a brain signal can be measured. The complexity of these data forces researchers to collapse them in some meaningful way. Typically, this often results in either focusing on central tendency of a given time series within-subject (e.g., calculating voxel means for specific task blocks in a block design study in fMRI; taking the trial averaged evoked potential wave form in EEG), on calculating spectral power density (e.g., more typical for EEG and MEG), or on using functional connectivity methods (e.g., correlation-based or graph theory metrics; independent component analysis; dynamic causal modelling) to capture point-to-point relations between brain regions. Interestingly, the within-person variability of momentto-moment brain responses typically is either completely ignored or is attributed to various confounds that are deliberately minimized (in the name of improving signal-to-noise ratios). However, an increasing body of work directly examining within-subject brain signal variability has revealed a host of powerful, and in many cases unexpected, links to healthy brain function, task performance, development, and various clinical conditions. Our intention in this brief review is to promote the idea that researchers should directly consider signal variability as a within-subject measure of interest in neuroimaging research, and importantly, that variability is a functional property of the human brain. We begin by discussing how researchers in areas of neuroscience outside of neuroimaging have studied and characterized signal variability. We then summarize relevant neuroimaging progress by discussing studies that have examined moment-to-moment brain signal variability across various samples and experimental designs. Finally, we discuss several key issues with, and provide several suggestions for, conducting research on neuroimaging-based brain signal variability.

\section{Brain activity is variable, and variability is often functional}

Various subdisciplines (cellular, systems, behavioural) within neuroscience have long shown that the brain is inherently variable from moment to moment at every level of the nervous system, no matter how its function is measured (Faisal et al., 2008; Stein et al., 2005). However, exactly what this variability represents has remained unclear. Typically, many researchers intuitively conceive of variability as neural "noise," a nuisance factor that presumably interferes with the efficiency of neural processes. In contrast, in an excellent review several decades ago, Lawrence Pinneo (1966) argued that neural variability "is not merely noise" (p. 245); rather, it enables sensory discrimination and learning, and perhaps surprisingly, the stable and functional output of a neural system. Specifically, he noted that the "tonic" activity of the brain (fluctuating, ongoing activity) provides the substrate for effective neural function, a concept borrowed from Arduini (1963) that is now central to the pervasive study of the brain's default activity (Deco et al., 2011; Raichle, 2010; Raichle et al., 2001, 2007). "Phasic" or stimulus-driven activity represents a relatively small proportion of total activity, and is argued to operate on existing tonic activity to permit behaviours of interest, a conceptualization that reaches back at least to Lashley (1951) and to one of Sherrington's disciples (Brown, 1914). Appropriately, Arieli et al. (1996) eloquently noted that "the effect of a stimulus might be likened to the additional ripples caused by tossing a stone into a wavy sea" (p. 1863).
Many extant notions of why variability is not simply noise and can be highly functional extend beyond that of a focus on tonic and phasic activity. For example, connectionist and cellular research suggest that networks formed in the presence of greater noise are more robust to disruption, thus enhancing learning and environmental adaptation, and helping to maintain optimal performance (Basalyga and Salinas, 2006; Faisal et al., 2008). This robustness may result from noise actually reducing the average weight attributed to any one network node; thus, if one element of a network is compromised, the chances of network disruption remain relatively small. Others have suggested that substantial trial-to-trial brain variability derives from coherent spontaneous oscillations throughout the cortex (Fox et al., 2005; Laskaris et al., 2003; Nir et al., 2008; Varela et al., 2001). From this perspective, signal variability may reflect functional connectivity between regions, a topic that has recently been explored in neuroimaging (Mišić et al., 2011a; Vakorin et al., 2011). Further, stochastic resonance (SR) research suggests that adding moderate noise to neural systems can help enhance the detection of weak signals, allowing subthreshold neurons to fire and potentially enhancing neural synchrony across multiple sensory modalities (Basalyga and Salinas, 2006; Faisal et al., 2008; Li et al., 2006; Lugo et al., 2008; McDonnell and Abbott, 2009; Ward, 2003; Ward et al., 2006). When noise is too low (inability to detect weak signals) or too high (noise saturates the signal), neural processes remain suboptimal; as a result, SR phenomena are often represented along an inverted U-shaped curve. A broader and more generalizable term that does not depend on signal detection for definition (as SR does) is "stochastic facilitation," which describes a variety of computational, animal, and human studies in which neural computations simply prove more effective in the presence of stochastic, biologically relevant noise (McDonnell and Ward, 2011; for a brief discussion of possible points of intersection between stochastic facilitation and studies of in vivo brain signal variability, see Garrett et al., 2011a).

Signal variability can also reflect greater dynamic range (i.e., the range of possible responses to incoming stimuli). Greater dynamic range is generally beneficial to the adaptability and efficiency of neural systems because it permits a greater range of response to a greater range of stimuli. Interestingly, broad-scale dynamic range is best achieved when synaptic excitation and inhibition are well-balanced (Shew et al., 2009, 2011), producing a natural ebb and flow in the system. As a nonlinear dynamical system, the healthy brain functions at the "edge of criticality," an optimal balance between myriad possible metastable states, which evolves also over multiple time scales via a static set of structural connections (Deco et al., 2011, 2009; Ghosh et al., 2008; Honey et al., 2007, 2009; McIntosh et al., 2010). Self-organized criticality is often evidenced by the presence of power-law (e.g., $1 / f)$ structure, mono-/multifractal scaling, and neuronal avalanche (i.e., coordinated bursts of neural activity which exhibit powerlaw characteristics) behaviour (see Achard et al., 2008; Bak et al., 1987; Bassett et al., 2006; Beggs and Plenz, 2003; Beggs and Timme, 2012; Bullmore and Sporns, 2009; Ciuciu et al., 2012; Fraiman and Chialvo, 2012; Plenz and Thiagarajan, 2007; Shew et al., 2009, 2011; Suckling et al., 2008; Tagliazucchi et al., 2012; Yang et al., 2012). Remarkably, dynamic range, information capacity, and information transfer optimize when brain networks are at criticality, signal variability is optimal, and network synchrony is maximally variable (Shew et al., 2009, 2011; Shew and Plenz, 2013; Yang et al., 2012). When variability is lacking, there is also little capacity for the brain to explore its state space, yielding the potential for the system to remain rigidly in a single state (Deco et al., 2009; Deco and Jirsa, 2012). Without the continual, fluctuating and variable "hum" of tonic brain activity then, state-to-state transitions may prove more difficult (either spontaneously or when required). Overall, such findings suggest that signal variability may index 
and/or facilitate neural malleability and adaptability from moment to moment.

Approximations to Bayes optimality within the brain may also go hand in hand with signal variability (Beck et al., 2008; Knill and Pouget, 2004; Ma et al., 2006). In this context, "Bayes optimal" suggests that a population of neurons will generally select a response that approaches the theoretical optimum from a distribution of potential responses, given some stimulus input. If neurons fired in exactly the same manner every time a specific stimulus was encountered (i.e., purely deterministically), one would be less able to adapt to different circumstances that involve that same stimulus. Populations of neurons may effectively encode probability distributions of responses (i.e., a probabilistic population code) given the reliability of incoming signals. Based on the proximity of the stimulus to each neuron's preferred stimulus criteria, an optimal response can be chosen. Importantly, the brain may also combine information from stimuli of varying reliabilities (through a simple additive process that requires the presence of adequate neuronal variability) to optimize its responses (Ma et al., 2006). In general, this could result in reliable and adaptable neuronal firing in the presence of stimulus uncertainty.

At a related, yet higher level of abstraction, signal variability may represent itinerant (wandering) dynamics in complex, self-organizing neural systems. Friston et al. (2012) argue that a characteristic feature of the brain is its tendency to destroy its own fixed points (i.e., not settling in to any particular state; Friston, 2010; Friston and Ao, 2012), which is key for enabling and maintaining itinerancy. There are several prominent connectionist conceptualizations of how itinerancy emerges and manifests. For example, populations of relatively weak attractors in close proximity to one another (such as Milnor attractors) can enable "chaotic itinerancy;" given that no attractor dominates the attractor landscape in this scenario, a natural itinerant exploration of the neural state space emerges (Friston et al., 2012; van Leeuwen, 2008). Another conceptualization refers to "multi-stability and switching" in neural systems (see Jirsa et al., 1994). In such models, attractors are typically stronger than Milnor attractors, but the presence of noise drives the system from one attractor to the next across moments, revealing itinerancy at local and network levels. In either case, itinerant dynamics are natural and can be functional. Friston et al. (2012) argue that itinerancy is necessary during Bayesoptimal perception (and is a crucial element for long-term free energy minimization (see Friston, 2010)); the authors state: “... if neuronal activity represents the causes of sensory input, then it should represent uncertainty about those causes in a way that precludes overly confident representations. This means that neuronal responses to stimuli should retain an optimal degree of instability that allows them to explore alternative hypotheses about the causes of those stimuli" (p. 3). This sentiment is convergent with that of Pouget et al. noted above (Beck et al., 2008; Knill and Pouget, 2004; Ma et al., 2006). Relatedly, van Leeuwen (2008) noted that neural itinerancy can operate as a novelty detector, enables state space exploration, and permits learning by discovery. Importantly, and again in line with Pouget et al., van Leeuwen (2008) argues that wandering dynamics provide/represent a necessary level of system flexibility from moment to moment, allowing the system to converge on optimal responses despite imperfect stimuli. Because the brain cannot know exactly what it will encounter across moments, it would be ideal if it did not become locked into any particularly rigid response pattern, an inefficiency problem cleverly referred to by van Leeuwen as neural "overfitting" (p. 87).

In summary, a variety of prominent ideas and potential explanations (e.g., dynamic range, Bayesian optimization, itinerant dynamics) exist that attempt to explain why signal variability should exist in neural systems. It is important to note that these potential explanations are not mutually exclusive, and various scenarios are plausible in which more than one may come into play (e.g., dynamic range may be necessary for itinerant dynamics-enabled Bayes optimality (and free energy minimization); without enough dynamic range, the brain could not sample the environment broadly enough over time, precluding the development of Bayesoptimal regimes). Although there is much work to be done before agreement is reached on exactly what signal variability "is", this selective summary provides some context and justification for why moment-to-moment brain signal variability is not only ubiquitous at every level of the nervous system (Faisal et al., 2008), but is also a vital component of healthy neural function. As a result, this work helps provide a theoretical and empirical grounding for the focused examination of within-person variability in neuroimaging signals, to which we turn next.

\section{Within-subject brain signal variability measures reveal powerful and novel insights into human brain function}

Over the past several years, scientists have begun to use neuroimaging to examine temporal brain signal variability as an individual differences measure of interest across a variety of samples and paradigms. A selection of these studies that focus on human development, cognitive performance, and clinical comparisons are described below. However, prior to discussing this body of work, we will first define what we mean by "temporal variability" in neuroimaging signals, and what types of measures have been used to quantify it.

In the vast majority of applications, temporal signal variability reflects a direct measure of the magnitude of some aspect of variability from moment to moment in a neuroimaging time series. The simplest forms of such measures include variance (He, 2011), standard deviation (Garrett et al., 2010, 2013; Wutte et al., 2011), and mean square successive differences (Leo et al., 2012; SamanezLarkin et al., 2010). Each of these measures taps related forms of overall distributional width. SD is simply the square root transformation of variance. MSSD (Neumann et al., 1941) is akin to variance, except that instead of a single, static mean serving as an anchor point, each time point is compared to the immediately preceding time point when calculating sums of squares. Another commonly applied family of measures examines signal complexity, or temporal unpredictability. One such measure is multiscale entropy (MSE; Costa et al., 2002, 2005), which is a derivation of Shannon's entropy (Shannon, 1948) and Pincus' early calculations of approximate entropy (Pincus, 1991). The MSE algorithm calculates sample entropy (Richman and Moorman, 2000) across multiple increasingly coarse-grained time scales. Sample entropy of each coarse-grained time series serves as an index of signal complexity by evaluating the occurrence of repetitive patterns; low MSE values reflect more deterministic or regular time series, and high MSE values indicate more complex and information rich signals. A number of other related complexity-type measures have also been applied in past studies (e.g., Omega complexity, Lempel-Ziv complexity, Lyapunov exponent, neural complexity; see Takahashi, 2012).

Another conceptualization and application of signal variability is dimensionality (e.g., principal components analysis (PCA, McIntosh et al., 2008); correlation dimension (Grassberger and Procaccia, 1983)). The more dimensions required to capture the variance among trials in a given voxel/electrode/sensor, the more variability that exists between those trials. Thus, when trials differ greatly from one another, they are less likely to be captured in the same latent dimension. Finally, in the frequency domain (via Fourier transformation), signals are represented as a sum of sinusoids, and the calculation of power (or spectral density) provides an index of the amplitude of sinusoidal oscillations within and across frequencies over a time series. In this way, power can serve as a specific 
form of signal variability. Recent fMRI studies have examined the amplitude (or fractional amplitude) of low frequency fluctuations (ALFF; Zou et al., 2008; Zuo et al., 2010) as a measure of resting state fluctuations, which is equal to taking the square root of the power in a particular frequency range. Relatedly, wavelet transformations allow measures of power/energy that can be calculated for more specific frequencies, wave forms, or time windows.

With various measures of signal variability in mind, we now highlight a series of recent applications of signal variability measures across samples and task types.

\subsection{Childhood to young adulthood}

Pre-adulthood neural development is characterized by multiple processes that could impact signal variability levels, including the maturation of neurotransmitter systems, pruning, myelination, and functional network change (Fair et al., 2009; Hasan et al., 2007; Johnson, 2001; Li, 2012; Li et al., 2010; Power et al., 2010; Sowell et al., 2003, 2004; Supekar et al., 2009; Toga et al., 2006; Uddin et al., 2011; Westlye et al., 2010). In particular, development yields increasing global (relative to local) network integration (Fair et al., 2009; Supekar et al., 2009), which could enable a balanced profile of neural differentiation and specialization coinciding with heightened process simultaneity, information capacity, and signal variability/complexity (Mišić et al., 2010; Tononi, 1998; Tononi et al., 1994). However, the study of developmental changes in brain signal variability per se (and relations with cognition and network function), have only recently been explored with neuroimaging.

In one study, McIntosh et al. (2008) examined relations between signal variability, age, and behaviour using EEG in a cross-sectional sample of 8-15 and 20-33 year olds on a face recognition task. The authors found that signal variability (whether measured using PCA or MSE) was higher in the young adult group compared to children and adolescents (see Fig. 1a), and was associated with higher face recognition accuracy and more consistent reaction time responses (reliable $R^{2}$ values ranged from 0.3 to 0.7 ). Comparing even younger children (1-66 months old) to adult controls, Lippe et al. (2009) examined EEG-based MSE during basic visual (black and white checkerboard stimuli) and auditory ( $50 \mathrm{~ms}$ broad band noise) stimulation. As in McIntosh et al. (2008), results indicated that MSE was greater with increasing age in both conditions (when modeled with continuous age, $R^{2} S=\sim .36$ ). Interestingly, visual (higher MSE) and auditory (lower MSE) conditions were differentiable in the younger groups, but diminished with maturation and were indistinguishable in adults. The researchers suggested this effect could reflect slower maturation (and possibly lesser functional integration) in lobes critical for auditory processing (temporal and prefrontal cortices) at early ages, relative to those regions more important for visual processing (Redcay et al., 2007; Sowell et al., 2004).

In another comparison of children/adolescents (6-16 years) and adults (20-41 years) Mišić and colleagues (2010) examined MEGbased MSE on a one-back working memory task involving face stimuli. One block of trials contained upright faces and the other contained inverted faces. Consistent with McIntosh et al. (2008) and Lippe et al. (2009), the authors found greater MSE across the cortex with maturation. This effect was most prominent in the posterior cingulate/precuneus for both task conditions. The upright face condition was generally distinguished by higher variability than the inverted face condition, primarily in the fusiform gyrus and anterior cerebellum. As in Lippe et al. (2009), the difference in MSE between conditions decreased with age; thus, adults showed greater signal variability overall, but this level of variability was more consistent across conditions. Finally, higher MSE correlated with higher accuracy, faster mean RT, and lower RT variability (see Fig. 1b for magnitudes). The authors concluded that greater variability during the upright compared to the inverted face condition may be attributable to naturally increased configural, holistic, and integrative processing present when viewing faces in their natural orientation. Thus, the authors present evidence for the sensitivity of MSE in distinguishing stages of development and task conditions.

Links have also been made between signal variability and functional connectivity. In a re-examination of the data from Lippe et al. (2009) noted above, Vakorin et al. (2011) explored the extent to which developmental increases in signal variability reflected either the localized specialization of network nodes, or the heightened integration between distal regions. To address this question, the authors decomposed total signal variability at individual EEG channels into "local" (variability near the electrode site) and "distributed" entropy sources (i.e., variability as a result of the mutual information between a given electrode and other electrodes), and then gauged how these sources varied with development. Results indicated that development was marked by a decrease in local entropy and an increase in distributed entropy, thus supporting an "integration" model of heightened signal variability with maturation from 1-66 months of age. Mišić et al. (2011b) expanded on the link between network connectivity and signal variability by examining EEG-based functional connectivity metrics (using graph theory) and MSE in a sample of ten-year-olds. The authors focused on the centrality of each network node with respect to the number of connections it made ("degree"), the ease with which the node could be reached from other nodes in the network ("efficiency"), and the tendency of the node to occupy a position on the shortest paths between other pairs of nodes in the network ("betweenness"). All three graph metrics positively correlated strongly $\left(R^{2}=.45\right.$ to .72$)$ with MSE across multiple time scales and brain regions (see Fig. 1c for a snapshot); thus, the greater the signal complexity in a given node, the greater its network centrality.

These studies support the utility of examining EEG- and MEGbased signal variability development across children, adolescents, and young adults. Across studies, greater signal variability is associated with maturation and better cognitive performance, distinguishes different cognitive conditions and stimulus types, and reflects the development of network integration and centrality.

\subsection{Aging}

A series of recent studies has also begun to examine differences in within-person brain signal variability across adulthood and into old age. Like early development, human aging is an excellent context for investigating signal variability. The concepts of "noisy" and inefficient processing in adult aging were initially discussed between the 1960s and the 1980s (Cremer and Zeef, 1987; Salthouse and Lichty, 1985; Welford, 1981, 1965). Human and computational modeling evidence (Li et al., 2001; Macdonald et al., 2009) suggest that various neural processing inefficiencies associated with older age reflect degradations in neurotransmission (Bäckman et al., 2006) and the integrity of white and grey matter (Raz, 2005; Raz and Rodrigue, 2006), and functional network change (e.g., Andrews-Hanna et al., 2007; Grady, 2012; Grady et al., 2010). However, until recently, the notion of age-related neural noise has not been formally tested in vivo by examining withinsubject brain signal variability/noise directly.

Within a sample of young and older adults, Garrett et al. (2010) investigated the presence of age differences in fMRI-based blood oxygen level-dependent (BOLD) signal variability during fixation blocks. To measure signal variability in each person and in each voxel, the authors calculated a modified time series standard deviation ( $\mathrm{SD}_{\mathrm{BOLD}}$ ) following a series of preprocessing steps (e.g., block normalization to limit variance overestimation due to low frequency drifts). The authors then compared standard deviation(SD)and typical mean-based spatial patterns on their zero-order and partialled age-predictive effects. Results indicated that not only 
A

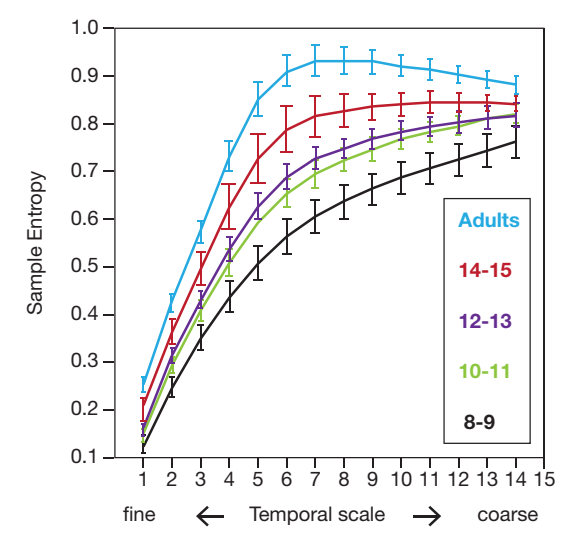

B

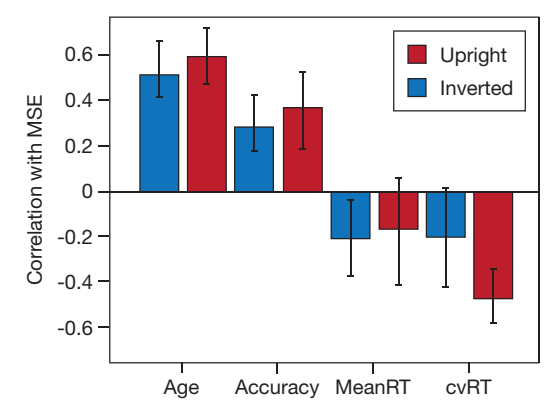

C
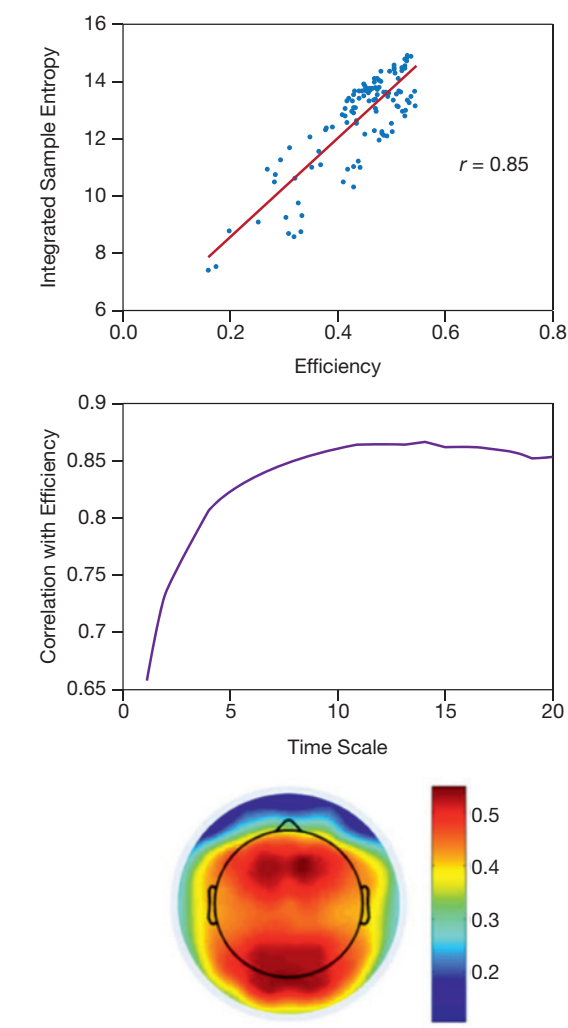

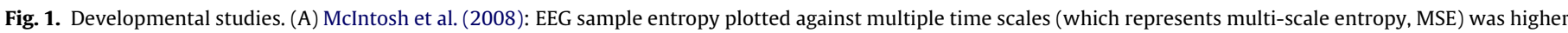

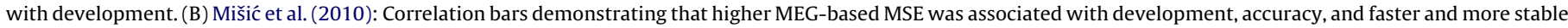

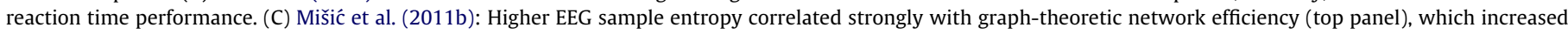
with coarse-graining of time series (middle panel), and was exhibited prominently over anterior and posterior electrodes (bottom panel).

was the $\mathrm{SD}_{\mathrm{BOLD}}$ pattern robust $\left(R^{2}=.81\right)$, this pattern was virtually orthogonal to that determined using mean BOLD signal, thus revealing a distinctive subset of age-relevant brain regions that would not have been discovered with mean-based measures. Notably, the unique age-predictive power of the $\mathrm{SD}_{\mathrm{BoLD}}$ pattern (unique $R^{2}=.27$ ) was more than five times that of the mean-based pattern (unique $R^{2}=.05$ ). Critically, contrary to earlier notions of age-related neural noise, but in line with the child-developmental studies noted above, younger adult brains were generally more variable overall than older adult brains, particularly in cortical regions.

In addition to age differences, associations between variability and performance emerged as well. In a subsequent study, Garrett and colleagues (Garrett et al., 2011b) examined task-related SD $\mathrm{BOLD}_{\mathrm{B}}$ in relation to age, and reaction time (RT) speed and consistency in younger and older adults on three cognitive tasks (perceptual matching, attentional cueing, and delayed match-to-sample). Multivariate models revealed that younger, faster, and more consistent performers exhibited significantly higher signal variability on all three tasks ( $R^{2}$ values ranged from $\sim .20$ to .65 ; see Fig. $2 \mathrm{a}$ ). Thus, beyond age, it appears that signal variability indexes individual differences in behavioural efficacy in older samples. As in Garrett et al. (2010), SD $\mathrm{BOLD}_{\mathrm{B}}$ and mean-based spatial patterns were essentially orthogonal, any regions that did overlap were largely opposite in directionality of effect, and mean ${ }_{\text {BOLD }}$ relations to age and performance were generally weaker $\left(R^{2}\right.$ values ranged from $\sim .10$ to .36$)$. Interestingly, younger, better performing adults not only presented with greater signal variability overall, but they also exhibited a greater range of variability levels across regions; older, poorer performers' variability levels were far less differentiated from region to region. In concert with the findings by McIntosh et al. (2008), Lippe et al. (2009) and Mišić et al. (2010), the Garrett et al. results (2010, 2011) provide preliminary support for an inverted u-shaped lifespan developmental curve of brain signal variability from infancy (lower variability) to young adulthood (high variability) to older adulthood (lower variability), as well as a generally monotonic and positive association between signal variability and cognitive performance.

Using a different set of analysis methods and functional tasks, another study (Samanez-Larkin et al., 2010) examined BOLD mean square successive difference $\left(\text { MSSD }_{\text {BOLD }}\right)^{1}$ values for each voxel in younger, middle-aged, and older adults across the entire time series of a financial decision task. Results of this study indicated that MSSD $_{\mathrm{BOLD}}$ was greater with increasing age, primarily in a number of subcortical regions (e.g., right caudate nucleus, right thalamus, midbrain; see Fig. 2b). Although age was associated with increasing variability, again there was a relationship between MSSD and task performance. Specifically, higher variability in the nucleus accumbens (NAcc) correlated with suboptimal financial decisions, and this relationship between NAcc variability and performance reliably mediated the zero-order effect between age and performance (see Fig. 2c for depiction and path coefficients). Surprisingly, in this task the authors found no brain regions that decreased in variability with age. It is worth noting that the Garrett et al. (2010, 2011b) studies also identified a few subcortical regions that exhibited greater

\footnotetext{
${ }^{1}$ It is noteworthy that $\mathrm{SD}_{\mathrm{BOLD}}$ (which is preceded by block normalization to minimize low frequency drift effects on variance calculations) was found to be statistically redundant with MSSD (Garrett et al., 2011b), thus allowing these measures to be directly compared.
} 
A
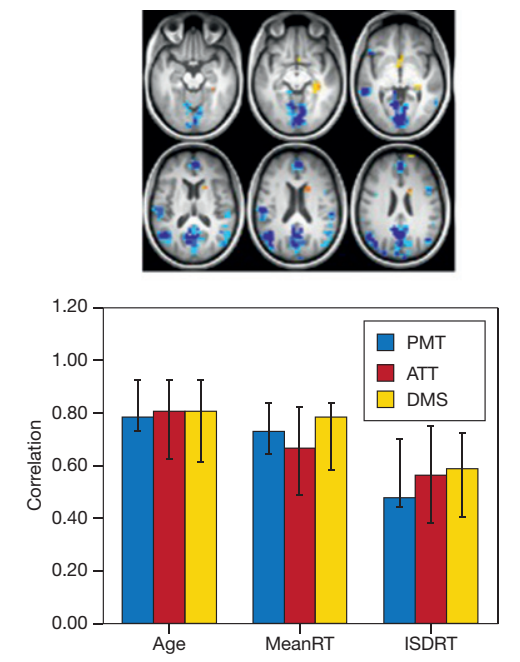

D

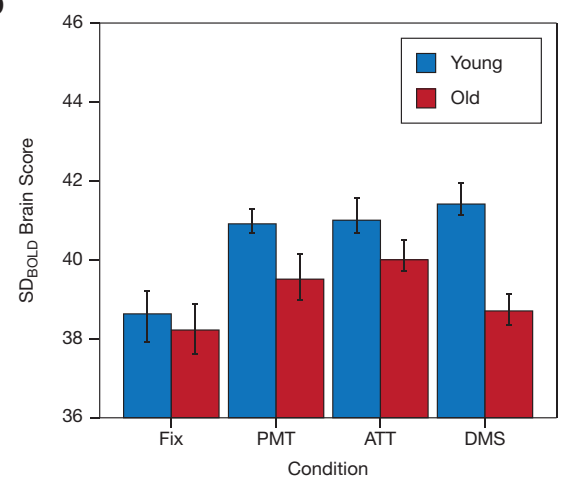

B

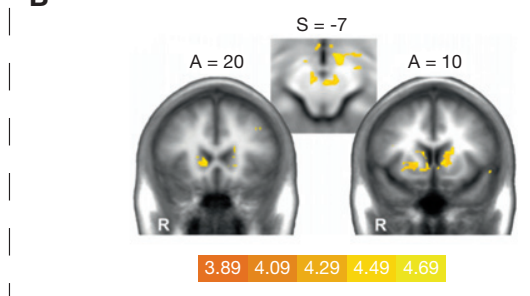

C
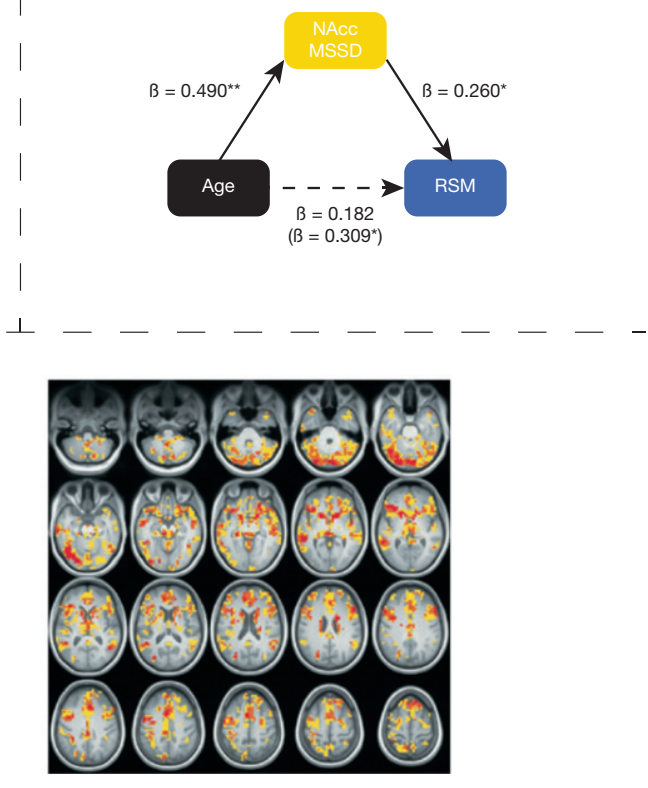

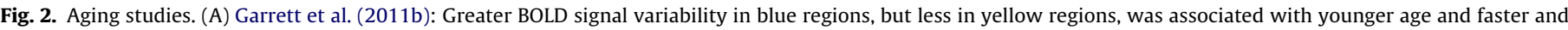

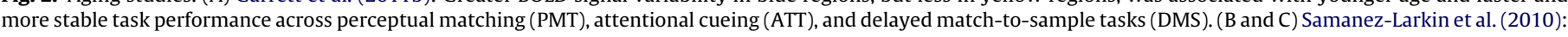

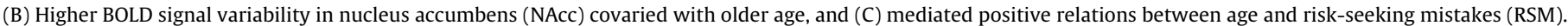
(D) Garrett et al. (2013): Younger adults were characterized by greater increases in BOLD variability from fixation to task (PMT, ATT, DMS), in yellow/red regions.

variability in older, slower, more inconsistent participants, convergent with the regions noted by Samanez-Larkin et al. (e.g., striatum, midbrain). The Garrett et al. and Samanez-Larkin et al. studies therefore suggest some consistency of findings at the subcortical level. Future research should address differences between subcortical and cortical signal variability effects, directionality of effects, and task types. Another point of convergence with Garrett et al. was that, for Samanez-Larkin et al., MSSD BOLD $_{\text {produced results highly }}$ differentiated from mean BOLD $_{\text {(i.e., mean }}$ BOLD produced no effects similar to $\mathrm{MSSD}_{\mathrm{BOLD}}$ ), thus further supporting the complementary information offered by signal variability measures.

Recently, Garrett et al. (Garrett et al., 2013) investigated the relationship between signal variability and changing cognitive demands. Within a sample of healthy younger and older adults, the authors found that $\mathrm{SD}_{\mathrm{BOLD}}$ was an effective discriminator between fixation and external cognitive demand (using the same three cognitive tasks as in Garrett et al., 2011b). Across a number of regions, signal variability increased broadly on task compared to fixation, particularly in younger and faster performing adults (interaction partial $\eta^{2}=.24$; see Fig. 2d). Conversely, older and slower performing adults exhibited fewer changes in signal variability within and across experimental conditions and brain regions, indicating a reduction in variability-based neural specificity. The authors argued that increases in signal variability on task may represent a more complex neural system capable of greater dynamic range between brain states, as well as an enhanced ability to efficiently process varying and unexpected external stimuli. Remarkably, despite sensitivity to a fixation versus task effect, $S_{B}{ }_{B O L D}$ revealed a unidirectional spatial pattern (broad scale increases in $\mathrm{SD}_{\mathrm{BOLD}}$ from fixation to task) entirely dissimilar to a prototypical "task negative" (at fixation) vs. "task positive" spatial pattern that one would expect when using mean BOLD $_{\text {as }}$ as the brain measure of interest.

MSE of brain signals has also been examined in relation to aging. For example, convergent with Garrett et al. (2013), one study (Takahashi et al., 2009) of younger and older adults noted that for young adults, EEG-based MSE values increased after a photic (light) stimulus was administered (relative to MSE levels immediately prior to stimulus onset) for $12 / 14$ electrodes. Older adults did not exhibit different MSE levels across tasks for any electrode. The authors' results suggest an aging-related loss of complexity and diminished functional response to visual stimuli.

\subsection{Healthy young adults}

Studies of signal variability in young adult-only samples have been scarce to date. One study (Wutte et al., 2011) investigated fMRI signal variability in the human motion complex (hMT+) during rest and during a visual discrimination task. Higher levels of fMRI signal variability on task compared to rest correlated with lower discrimination thresholds $\left(R^{2}=.36\right.$; see Fig. 3a), indicating that more variance during task is functional in the presence of environmental demand (Garrett et al., 2013; Takahashi et al., 2009). The 
authors' result is also consistent with stochastic resonance theory, which suggests that endogenous or exogenous noise/variability can increase the sensitivity of neuronal populations to incoming signals. In another study, He (2011) examined fMRI variance from 21 regions of interest (ROIs) in a series of resting and task runs (subjects were asked to press a single button when a crosshair dimmed onscreen), each of which were seven minutes long. Divergent from Garrett et al. (2013) and Takahashi et al. (2009), He found that variance decreased from fixation to task in 11 of 21 ROIs. However, the author's results also indicated that signal variance is positively correlated with scale-free signal properties $\left(R^{2}=.27\right.$; see Fig. 3b), suggesting that systems with higher signal variability may be operating nearer to a critical state. Ongoing young-only work from our group (Garrett et al., under review) suggests that the directional shift (up/down) in signal variability from fixation to task depends on the exact parametric level of task difficulty for a given participant. This new work may help address divergent findings between $\mathrm{He}$ (2011) and other rest versus task studies reviewed above (Garrett et al., 2013; Takahashi et al., 2009; Wutte et al., 2011).

Mennes et al. (2011) explored relations between fMRI-based resting-state fractional ALFF and task-related mean activity and cognitive performance during an Ericksen flanker task. The authors found that fractional ALFF measures positively predicted taskevoked activity, but only in a small subset of task-active regions. This general finding agrees with previous work (Garrett et al., 2010; Garrett et al., 2011b) highlighting the occasional, but generally absent, relation between signal variances and means. Convergent

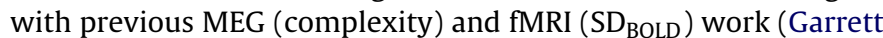
et al., 2011b; Mišić et al., 2010; Raja Beharelle et al., 2012), the authors also found that greater precuneus fractional ALFF (among other regions such as anterior and paracingulate cortices, insula, middle and orbitofrontal cortices) correlated with faster and more consistent reaction time performance. Conversely, and in line with Garrett et al's. (2011) aging results, cerebellar dominant clusters (but also including lateral occipital cortex, and postcentral, inferior temporal, orbitofrontal and fusiform gyri) exhibited the opposite direction of effect, in which greater fractional ALFF covaried with slower and less consistent performance. Here in young adults, as in older adult studies (e.g., Garrett et al., 2010; Garrett et al., 2011b; Samanez-Larkin et al., 2010), the nature and purpose of regionally specific links to performance require careful future examination and theoretical development.

Finally, a recent study expanded the study of signal variability into the domain of learning and memory. Heisz and colleagues (Heisz et al., 2012) investigated the effects of face familiarity,
A

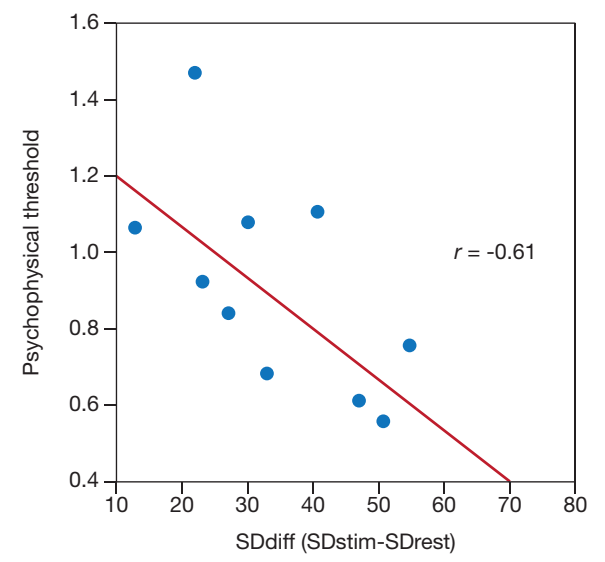

B

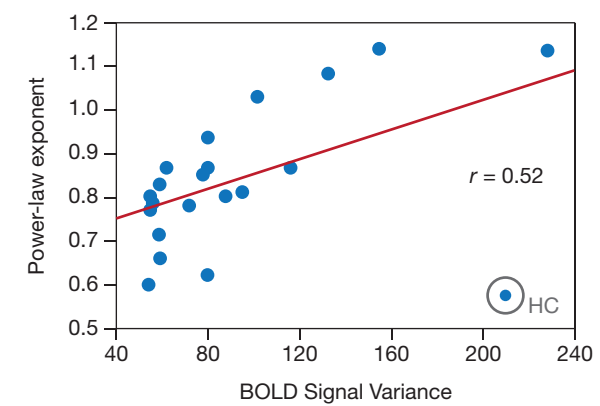

I

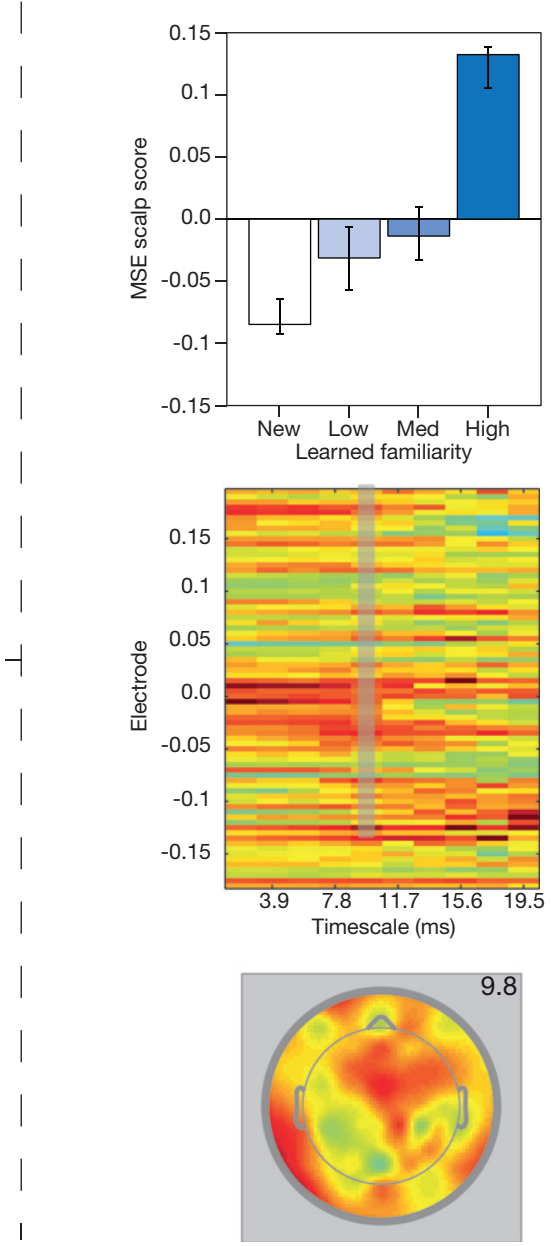

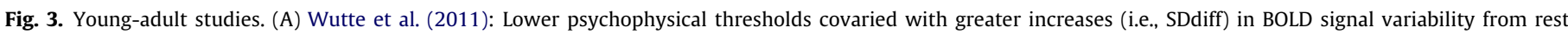

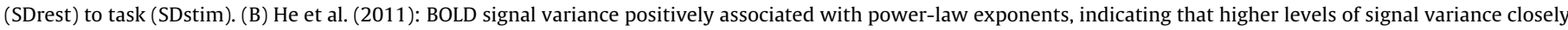

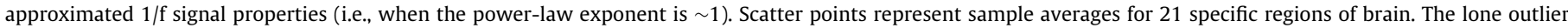

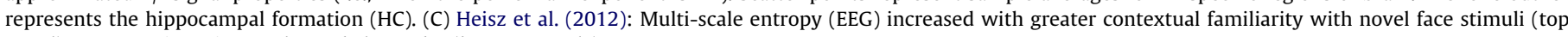
panel), across various time scales and electrodes (bottom panels). 
repetition, and learning on MSE levels. Results indicated that higher subject ratings of famous face familiarity correlated with higher MSE values across electrodes. Within-person, higher MSE levels also covaried with greater experimental exposure to previously unknown faces that were paired with audible stories (see Fig. 3c). The authors argued that cognitive processes during the perception of familiar stimuli may engage a broader network of regions manifested as higher complexity/variability in spatial and temporal domains.

These various young adult studies demonstrate that even in relatively homogeneous, healthy samples, signal variability methods can be highly sensitive indicators of task conditions and cognitive performance.

\subsection{Disease and brain injury}

To the extent that brain signal variability is an index of neural dynamics, complexity, and functional performance, the utility and sensitivity of signal variability measures should extend beyond healthy samples, and serve as an important marker of individual differences for clinical comparisons. Several years of notable work on dementia, schizophrenia, traumatic brain injury (TBI), autism, congenital blindness, mesial temporal lobe epilepsy, and other conditions suggests this is indeed the case. In particular, the study of complexity, rather than variance, has permeated these areas of research. Depending on the nature of the pathology, available studies have been more or less mixed with regard to whether greater signal variability characterizes healthy controls or not.

Dementia (particularly Alzheimer's Disease, AD) and schizophrenia have received a large proportion of past research attention on signal variability/complexity. These studies have largely focused on resting state EEG/MEG data, using various forms of complexity measures. Regarding AD, most studies examining single temporal scales to estimate complexity (see Dauwels et al., 2010; Stam, 2005; Takahashi, 2012) find that AD patients exhibit less signal complexity than healthy, age-matched controls (although BOLD-based signal variability findings in mild cognitive impairment are mixed, see Han et al., 2011; Xi et al., 2012). Some multi-scale findings further support this group difference across time scales (Escudero et al., 2006). However, other multi-scale findings invite different conclusions. For example, Park et al. (2007) demonstrated an MSE-based reduction in complexity in severe $\mathrm{AD}$ at finer time scales, but the opposite effect tended to occur at coarser time scales (severe AD signal complexity was higher at slower frequencies). A recent EEG study (Mizuno et al., 2010) corroborated these effects. AD patients (including mild to severe cases) had lower signal complexity (MSE) at finer scales in frontal, temporal, and parietal electrodes. However, when comparing severe $\mathrm{AD}$ cases to controls, patients exhibited higher MSE at coarser scales across electrodes. These findings demonstrate the power of a multi-scale approach to quantifying signal complexity. In both fine and coarse-grained time series, AD appears reliably discriminable from healthy adults, albeit in opposite ways.

Similar to the bulk of AD findings, the current state of findings regarding schizophrenia have been mixed, with at least as many current studies noting greater (e.g., Hoptman et al., 2010) as noting lesser signal variability in schizophrenics (see Fernandez et al., 2012). However, Fernandez et al. (2012) propose that the observed discrepancies may be somewhat reconcilable; when all available evidence is considered, young, medication-naïve, symptomatic schizophrenics are most likely to exhibit increased signal complexity relative to healthy controls. The apparent consistency of this effect may designate schizophrenia as a special case in the study of complexity, within which the diseased group's brain signals appear more complex. Similar to AD research though, multiscale approaches have been underutilized in past schizophrenia research, with notable exceptions that highlight frequency band specificity of complexity effects (Takahashi, 2012; Takahashi et al., 2010). In any case, no matter the direction, biocomplexity and variability measures have been highly powerful discriminators of schizophrenics and healthy controls.

The study of other clinical populations is much clearer in its directionality, revealing that healthy brains are more variable and complex. Multi-scale work on TBI (Raja Beharelle et al., 2012) investigated relations between MEG-based MSE during an attention task in healthy adults and patients with TBI. Lower MSE values characterized TBI patients compared to controls (see Fig. 4a). Higher MSE also predicted more accurate and consistent performance for healthy adults $\left(R^{2}=\sim .25\right)$, and more consistent performance for TBI patients $\left(R^{2}=\sim .50\right)$. Interestingly, the relation between MSE and performance consistency was stronger for people with brain injury, and depended on specific condition (more difficult conditions revealed stronger relations) and MSE time scale (the moderately difficult condition revealed the effect at finer time scales; the most challenging condition revealed the effect at coarser time scales). Nenadovic et al. (2008) also examined the EEG-based temporal variability of phase synchronization in a sample of children who had experienced a TBI and resulting coma, and compared them to age- and gender-matched controls. The authors examined whether the variability of EEG phase synchrony (between electrodes), analyzed during the acute phase post-TBI, would differ from that of normal children. Brain activity was measured during an eyes-closed condition. The authors found that greater temporal variability of phase synchronization among EEG electrodes strongly covaried with greater recovery and emergence from coma twelve months after TBI $\left(R^{2}=.55\right)$. Interestingly, phase synchronization variability was highly similar between normal controls and those patients who eventually recovered well. The authors suggested that variability of phase synchrony may offer a valuable predictor of current functional integrity (convergent with computational and animal work; Yang et al., 2012), as well as future functional outcome in brain-damaged patients.

An interesting application of fMRI signal variability measures was recently reported in a small sample comparison of congenitally blind and healthy adults (Leo et al., 2012). Congenital blindness provides an interesting context within which to examine signal variability. On one hand, from a perspective of biocomplexity, one might expect healthy adults to have more complex systems given the need to consistently process and integrate information from greater, rather than fewer, sensory sources. However, previous work suggests that the life-long absence of retinal input can induce cross-modal plastic reorganization in early visual brain areas, which then process stimuli conveyed by auditory, tactile, and olfactory senses, thus characterizing such regions by their "supramodal" and information-rich status. The authors hypothesized that parietal cortex may be an obvious hub region to transmit multi-modal information to visual cortex. On two different tactile tasks (spatial discrimination and motion perception), the authors found that blind individuals exhibited greater signal variability (using $\mathrm{MSSD}_{\mathrm{BOLD}}$ ) across parietal, occipital, temporal, and frontal regions (see Fig. 4b). Using an inferior parietal seed region, functional connectivity analyses also supported positive relations with superior and middle occipital regions (among others), and showed greater connectivity for the blind group. Although a larger sample and the inclusion of task performance data would have added greatly to conclusions made, this study provides a good example of the potential sensitivity of signal variability measures to the effects of cortical plasticity and supramodal region development.

New multi-scale research on EEG signal complexity in autism is emerging. One group (Bosl et al., 2011) investigated group differences in resting-state MSE in normal developing children and children at high risk for autism spectrum disorder. Across all 
A

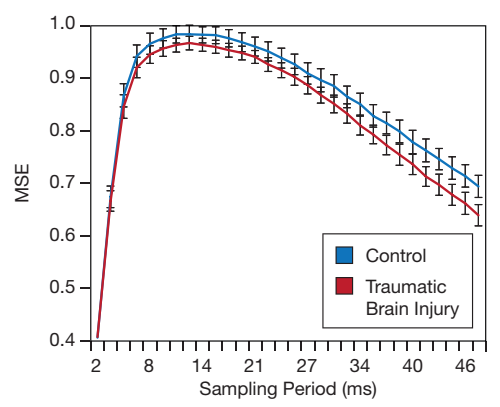

B

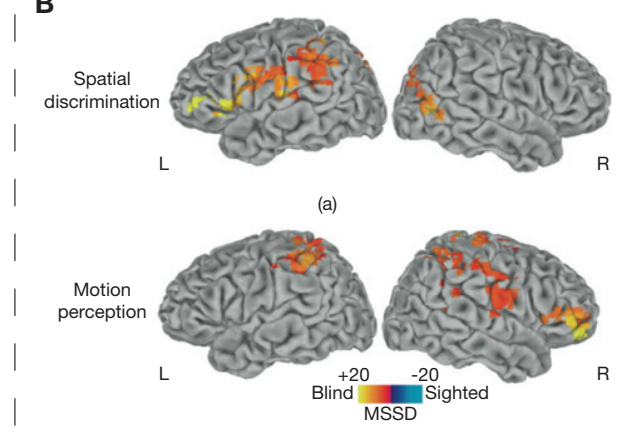

c
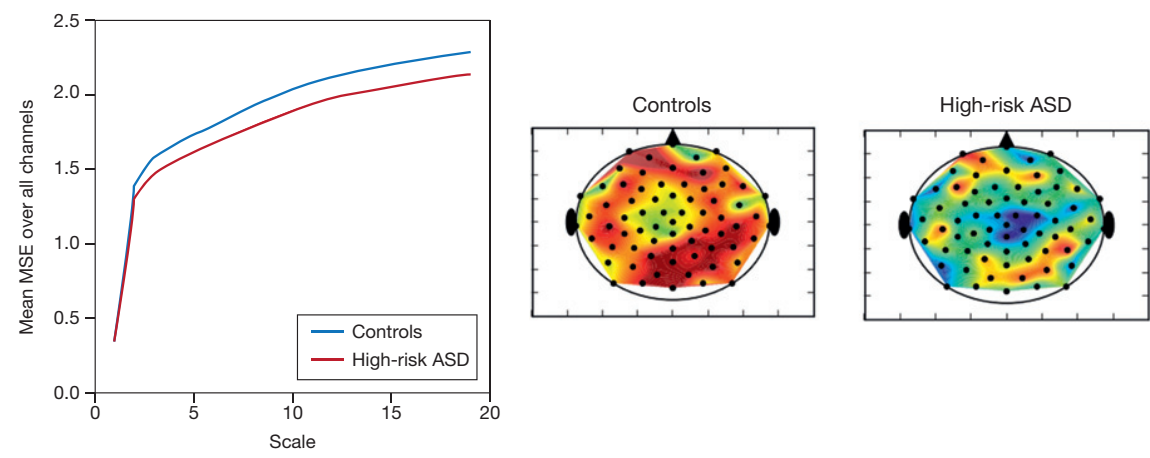

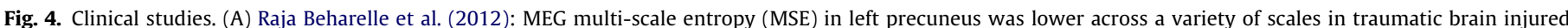

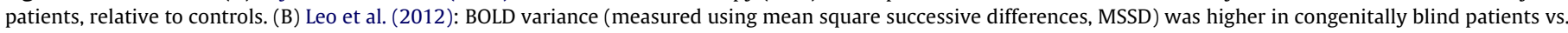

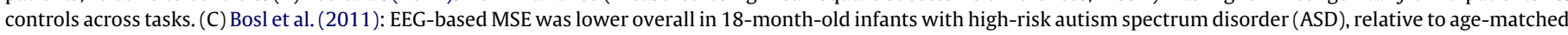
controls.

channels and the majority of temporal scales, MSE tended to be greater in normal children (see Fig. 4c). Using several machine learning algorithms with MSE as a feature vector, the authors correctly classified groups with $70-100 \%$ accuracy. Of the relatively few clinical studies to utilize task data, Caterino and colleagues (2011) examined MSE differences between normal and autism spectrum condition-diagnosed adults during face and chairmatching tasks. Results indicated lower MSE values in the autism group across temporoparietal and occipital regions for both tasks, particularly within coarser scales (i.e., lower frequency scales). Importantly, no significant differences in EEG power spectra were observed between groups, indicating that changes in complexity values are not necessarily a reflection of changes in EEG power spectra.

Epilepsy also continues to receive attention in the study of signal variability. Protzner et al. (2010) applied MSE measures to examine intracranial EEG-based MSE within a sample of mesial temporal lobe epilepsy patients while they performed a scene encoding and recognition task. Each patient presented with a history of right mesial temporal seizure onsets, and had electrodes implanted within the right (epileptogenic) and left (healthy) hippocampi. The healthy left hippocampus was marked by significantly higher MSE across temporal scales, especially during scene encoding. This suggests that cognitive demand can influence the sensitivity of signal variability to differentiate damaged regions from their healthy homologues. Other notable EEG work finds that although they may not differ in signal entropy across the brain relative to controls during normal periods (Burioka et al., 2005), epileptics are characterized by gross reductions in entropy in the minutes prior to, and during, seizures (Burioka et al., 2005; Zandi et al., 2009).

A number of other disorders and diseases have also been targets for variability and complexity analyses (Guo et al., 2012a,b;
Takahashi, 2012; Yu-Feng et al., 2007; Zhu et al., 2012), thus further supporting the use of these measures for studying group differences and clinical outcomes. However, the application of variability/complexity techniques in clinical neuroimaging remains in its infancy, with relatively few studies taking a multi-scale approach. As findings accumulate within and across clinical samples, stronger claims about directionality at particular frequencies can emerge.

\subsection{Summary}

The aforementioned studies of brain signal variability across the lifespan and in brain injury and disease reveal novel and powerful insights into brain development, behavioural correlates, and neural integrity. The cellular, animal, and neurocomputational foundations, predictive power, and complementary nature of signal variability suggest that methods focusing on variability will continue to bear fruit in human neuroimaging research. Although there is a great of deal of work required to understand what the various variability and complexity measures tell us about brain function, these measures are remarkable in their ability to provide a unique window into the human brain that typical brain signal analytic methods do not provide.

\section{Potential issues, preliminary solutions, and future directions}

With the emergence of signal variability research as a potential next frontier in brain imaging, it is becoming vital that scientists work towards best practices. As a first step, we attempt to highlight several key points and possible solutions we think are important at this juncture in the field, and of which anyone doing this research 
may wish to be aware. We also describe various next steps and opportunities for expansion in this broad area of research.

\subsection{Clearly define your desired level of signal variability measurement precision}

Beyond measure type (e.g., complexity, variance), momentto-moment temporal signal variability can take on many forms. For example, in any imaging paradigm, depending on researcher interests and available temporal resolution, one may wish to characterize single-trial variability within a given condition (McIntosh et al., 2008), measure the variability across trials within condition (Garrett et al., 2010; Garrett et al., 2011b; Samanez-Larkin et al., 2010), compare variability levels between conditions (Garrett et al., 2013; He, 2011; Mišić et al., 2010; Wutte et al., 2011), or compute variability across a number of conditions or states. It is not yet known how these different levels of variability analysis relate, which may be a potential source of between-study differences in directionality or power of research findings. If temporal and/or spatial resolution allow, it would be worthwhile to examine as many of these levels of precision as possible in a given study, so that readers can appreciate how signal variability may differ at various levels of data aggregation.

Relatedly, optimal task design should ensure that signal variability can be accurately measured at one's preferred level of precision. In fMRI for example, $\mathrm{SD}_{\mathrm{BOLD}}$ levels respond to specific task types, perhaps representing different variability-based "states" (Garrett et al., 2013). However, event-related-type fMRI designs that iterate through multiple successive trial types may be less accurate at capturing within-state signal variability that is uncontaminated by other states. In such cases, it is possible that the brain establishes a more balanced level of signal variability to handle the varying load under which it is placed. This could be experimentally verified. For electrophysiology (EEG/iEEG/MEG/ECoG), one indeed has ample temporal precision to examine variability at the single trial level, but this still does not ensure that state mixing has not occurred, to the extent that residual variance from a preceding trial can impact successive trials over particular time scales. As a result, the use of block designs may be better suited to the precise measurement of signal variability.

\subsection{Preprocessing is key}

When variability is the focal measure of brain function, preprocessing of data (and precise documentation of specific steps taken when publishing results) may become even more important than is typically the case. Various artifacts (e.g., in fMRI, extreme spikes due to head motion, drift artifacts, signal/slice drop-out; in EEG, eye, muscle, cardiac movements) can have profound impacts on the statistical power and reliability of variability measures. In fMRI, extended preprocessing pipelines (i.e., beyond typical spatial normalization, smoothing, slice time correction, and motion correction) that include intra-individual ICA denoising, $\mathrm{WM} / \mathrm{CSF} /$ motion parameter regression, and block normalization have been shown to simultaneously reduce average voxel variance levels, while dramatically increasing statistical prediction (Garrett et al., 2010). Although much needs to be done (e.g., examination of whether fMRI signal variability results should be scaled by responsivity measures such as ASL or hypercapnia), Garrett et al's. (2010) results suggest that by progressively honing brain signals via principled preprocessing, we can obtain more reliable signal variance sources (for within- and between-session reliability of BOLD signal variability, see Garrett et al., 2013; Zuo et al., 2010). Encouragingly, systematic sources of artefact-related variance in BOLD signals often account for a relatively small proportion of the variance that exists in BOLD time series data anyway; the majority of signal variance likely represents the variability of actual neural responses (Bianciardi et al., 2009; Kruger and Glover, 2001), thus supporting the continued exploration of variability as an important measure of brain function.

\subsection{Statistical methods of choice need not change, but what about calculating signal variability?}

The choice to examine brain signal variability is essentially orthogonal to which models can be used to analyze it. Because statistical models/packages are blind to the various sources/types of data, all that is required is for signal variability data to be in the correct format for it to be analyzable in a standard manner (e.g., general linear model; multivariate frameworks such as PLS or MVPA). For example, our SD $_{\text {BOLD }}$ models (Garrett et al., 2010, 2013; Garrett et al., 2011b) were run wholly within the PLS software package for MATLAB, requiring only that the input data matrix represent voxel SDs rather than voxel means. Similarly, after proper formatting (e.g., using 3dcalc in AFNI), standard AFNI regression models (3dregana; Cox, 1996) were used to examine Samanez-Larkin et

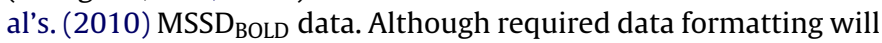
vary by analysis package, the time taken to format thus far has been reasonable in our own studies.

Unfortunately, the primary barrier to any widespread adoption of signal variability measures is that no major imaging software package (e.g., SPM, FSL, AFNI, EEGLAB) currently has comprehensive signal variability estimation tools built-in. To our knowledge, outside of the readily available estimation of power/fluctuation amplitudes (e.g., ALFF measures available via the REST toolbox (Song et al., 2011); wavelet and Fourier-domain analyses in EEGLAB (Delorme and Makeig, 2004); MATLAB's (Mathworks, Inc.) Signal Processing or Wavelet Toolboxes; FSL's fslspec tool; AFNI's 3dPeriodogram or 3dWavelets tools), little else is easily accessible. In our work, we have adapted (e.g., Costa et al.'s MSE scripts; http://www.physionet.org/physiotools/mse/tutorial/) and have created various scripts within MATLAB or Unix shell environments (e.g., for $\mathrm{MSSD}_{\mathrm{BOLD}}$ or block detrended $\mathrm{SD}_{\mathrm{BOLD}}$ measures), although other programming languages/environments (e.g., Python) would also be workable. We plan to make various tools/scripts we use freely available (hosted at douglasdgarrett.com, and eventually at nitrc.org), with a longer-term goal of developing comprehensive toolboxes for the major open-source image analysis packages. In the meantime, primary references for description/implementation of commonly applied brain signal variability measures are: (1) SD $_{\text {BOLD }}$ (Garrett et al., 2010, 2013; Garrett et al., 2011b); (2) MSSD $_{\text {BOLD }}$ (Leo et al., 2012; Samanez-Larkin et al., 2010); (3) ALFF/fALFF for BOLD signals (Zou et al., 2008; Zuo et al., 2010); (4) MSE (Costa et al., 2002, 2005; McIntosh et al., 2008), which is a multi-scale version of sample entropy (Richman and Moorman, 2000). As always, interested readers can seek out any number of excellent signal processing references that cover various aspects of fluctuation amplitudes, stochastic processes, and general signal dynamics (e.g., Mitra and Bokil, 2008; Schomer and Lopes da Silva, 2011).

\subsection{Linking variability and complexity/entropy metrics?}

Despite their continued use, it remains unclear how different measures of brain signal variability such as signal variance (e.g., $\left.\mathrm{SD}_{\mathrm{BOLD}}\right)$ and MSE relate. As noted above, MSE captures the complexity of point-to-point transitions in time series, rather than the overall variance in a time series. Because MSE is calculated across multiple time scales, substantial data are required to calculate stable estimates. Although MSE measures may be highly useful when applied to imaging techniques with high temporal resolution (EEG, MEG, fNIRS), many task-based fMRI studies are simply 
not amenable to their full implementation (although, the spatial resolution of fMRI is superior). In typical block design studies in cognitive neuroscience, condition blocks are too short (e.g., often $30 \mathrm{~s}$, repetition time (TR) often $=2 \mathrm{~s}$ ) to allow the estimation of multiple frequencies and temporal scales. Even if block concatenation was employed, the original time series would still be altered. The brevity of task blocks is often by design, however. To maximize subject attention and performance, and to avoid fatigue and neural adaptation effects, relatively short task blocks are typically offset with fixation/rest blocks. Studies designed to compute variability/complexity over very long continuous task blocks should work to ensure that variability is not compressing systematically over time, simply due to fatigue or neural adaptation. This is likely particularly important for work with populations who may be more or less susceptible to cognitive and or/neural adaptation and fatigue (e.g., older adults, diseased participants).

Even in the presence of study designs allowing for robust calculation of variance and entropy measures (e.g., resting-state data), these two measure types are not yet statistically comparable in their present forms. For example, most entropy measures (Richman and Moorman, 2000) directly scale away signal variance by setting the "similarity criterion" parameter (i.e., how one quantifies if two points in a time series are "the same" or not) as a percentage of signal variance. It remains a topic for future research what the solution to this problem could be. Even if not immediately comparable, entropy and signal variance continue to provide complementary and independently powerful information about brain function, and should be pursued further.

Finally, given the similarity/complementarity of findings between MSE and the spectral power distribution (Catarino et al., 2011; Heisz et al., 2012; Lippe et al., 2009; McIntosh et al., 2008; Mišić et al., 2010), it would be fruitful to continue the examination of the frequency domain in concert with variance and MSE measures (e.g., to see which measures best covary with age, performance, disease). In particular, various groups continue to examine measures of fluctuation amplitude in the frequency domain across multiple frequency bands (Zuo et al., 2010), and such metrics could easily be integrated into a comprehensive study of neuroimagingbased signal variability should enough continuous time data be available in experimental designs of interest. As amplitude fluctuations are one form of signal variance, this would provide another possibility for comparing the relative predictive utility of entropy and variability measures.

\subsection{Implications of signal variability for the study of functional connectivity}

The examination of links between signal variability and functional connectivity between regions is inherently interesting. The dynamics in any signal are a function of inputs and outputs, logically pushing signal variability work in this direction (Mišić et al., 2011b; Vakorin et al., 2011). However, many common functional connectivity metrics (e.g., Pearson $r$, mutual information/joint entropy) scale away variance in their calculation. Although variance normalization allows the relation between a pair of brain regions to be directly compared to another pair, it remains unclear how signal variance and functional connectivity estimation interact. As perhaps the most commonly applied measure of functional connectivity in neuroimaging today, consider the Pearson $r$ formula (see Zalesky et al., 2012):

$$
r=\frac{\sum(x-\bar{x})(y-\bar{y})}{\mathrm{SD}_{x} \mathrm{SD}_{y}}
$$

The covariance (i.e., the numerator) between two brain regions is scaled outright by the within-region signal variability (i.e., the denominator). Because of strong group and performancerelated differences in voxel variability (Garrett et al., 2010, 2013; Garrett et al., 2011b), the examination of links between signal variability and connectivity would benefit if future work verified whether covariances scale with voxel variance levels as expected, or whether either the numerator or denominator may instead be driving the correlation effect. This is likely even more important when group differences in connectivity levels are also sought, to the extent that group differences in voxel variability levels are already known to exist (Garrett et al., 2010, 2013; Garrett et al., 2011b).

\subsection{Interpreting spatial patterns in fMRI}

One key finding from the $\mathrm{SD}_{\mathrm{BOLD}}$ findings is that resulting spatial patterns are effectively orthogonal to those defined by the mean signal (Garrett et al., 2010; Garrett et al., 2011b). Although this is a clear vote for the complementary nature of variability measures in fMRI, this orthogonality simultaneously renders challenging the interpretation of those same spatial patterns. The preponderance of fMRI literature in cognitive neuroscience is based on mean BOLD $_{\text {B }}$ (whether univariate or multivariate methods are employed). As a result, variability-based spatial patterns should not be interpreted according to this same literature and logic. How do we proceed? We must first establish a baseline sense of where in the brain variancebased effects most often emerge (and under what conditions), which requires remapping cortical function at rest and on various tasks. Existing studies (e.g., Garrett et al., 2010, 2013; Garrett et al., 2011b; Samanez-Larkin et al., 2010) provide first attempts to establish the spatial form and function of BOLD variability under various experimental constraints. Using MEG, Mišić et al. (2010) also highlighted important regions that comprise signal variability effects; future work in this area could closely compare MSE spatial patterns to those determined using mean-based methods (e.g., average signals; evoked potentials).

This ultimate goal of mapping function according to variabilitybased metrics (and comparisons to mean signal-based patterns) requires much future work, but could be expedited through examining signal variability within widely available archival data (e.g., Human Connectome Project; Alzheimer's Disease Neuroimaging Initiative (ADNI); 1000 Functional Connectomes Project; International Neuroimaging Data-Sharing Initiative (INDI)). Further, no-cost, systems-level simulations of signal variability effects using fMRI, EEG, and MEG signal sources are now possible and available to the public via The Virtual Brain project (www.thevirtualbrain.org). For example, within The Virtual Brain, one could simulate how fMRI-based orthogonality between signal means and variances changes as a function of local and systems level parameters, by region/network, and across time. Beyond the obvious cost efficiency involved, the robustness of variability effects noted in the present review suggest that utilizing freely available data and simulation tools could be incredibly fruitful, and would propel the study of signal variability across samples and task domains.

\subsection{Linking within-person signal variability to other individual differences}

Regrettably, links between signal variability and key individual difference variables such as affect and personality have rarely been made in the literature. Sparsely available studies have examined fMRI-based fractional ALFF measures in relation to cognitive and affective empathy (Cox et al., 2012), and multiple aspects of mood (e.g., depression, anger, vigor) pre- and post-tryptophan depletion (Kunisato et al., 2011a,b) with mixed results. Regarding personality, one key point of interest may be the study of neuroticism. Neuroticism is typically argued to represent psychological "instability", is often associated with anxiety, and is correlated with more variable 
reaction time performance, highlighting various potential levels of processing inefficiency (e.g., Hagger-Johnson et al., 2012; Robinson and Tamir, 2005). Interestingly, in light of consistent negative relations between brain signal variability and reaction time variability across various samples and paradigms (Garrett et al., 2011b; McIntosh et al., 2008; Mišić et al., 2010; Raja Beharelle et al., 2012), it might be expected that neuroticism may covary with less, not more, signal variability. Young-adult evidence for this direction of effect now exists (Kunisato et al., 2011a,b), and could be examined further in studies linking also to cognitive performance variability. More generally, how broad-scale "psychological entropy" (Hirsh et al., 2012) is associated with neural entropy/variability would be an excellent topic for future research.

Studies of neurochemical modulation and neurogenetics are also logical individual differences targets to pursue in relation to brain signal variability. Although many different transmitter systems could be targeted, one particular area of broad-scale interest may be the dopamine system. In human aging research for example, various studies have focused on dopamine, and dopamine-related genetic influences such as catechol-O-methly transferase (COMT) over the past two decades (e.g., Bäckman et al., 2006, 2010; Li et al., 2001; Macdonald et al., 2009, 2006). Notably, D1 and D2 receptors degrade $\sim 5-10 \%$ per decade across adulthood across the midbrain, striatum, frontal lobe, hippocampus, amygdala, anterior cingulate, and other cortical regions (see Bäckman et al., 2006; Li et al., 2001). Neurocomputational models of dopamine degradation with age (Li et al., 2006, 2001) demonstrate that age-related dopamine loss can yield more inefficient stimulus discrimination, a lower average neuronal firing pattern, and a striking dedifferentiation of neural responses in the face of varying stimuli. Given that slower/less consistent cognitive performance and greater dedifferentiation characterize generalized aging-related reductions in signal variability (Garrett et al., 2013; e.g., Garrett et al., 2011b), it would be interesting to test whether DA-related neural inefficiency (measured through multimodal PET/fMRI, neurogenetics (e.g., COMT met vs. val allele carriers; (Mattay et al., 2003)), and/or through pharmacological imaging studies) manifests in differences in signal variability in vivo. We are currently acquiring and examining multiple data sets to these ends.

Overall, to the extent DA is considered a key agent subserving “signal fidelity" (Bäckman et al., 2010, 2006; Li et al., 2001) that provides a neurochemical basis for signal dynamics to emerge, the use of signal variability measures in any DA-relevant area of research is warranted (e.g., decision making/reward/learning/response vigor (Dayan and Walton, 2012; Doya, 2008; Montague et al., 2004; Niv et al., 2007; Niv and Schoenbaum, 2008); impulsivity/addiction (Bogdan et al., 2012; Hariri, 2009); working memory/cognitive control (Bäckman et al., 2010, 2006; Cools and D'Esposito, 2011); cognitive training (Bäckman et al., 2011; Bäckman et al., 2010)). The relevance of signal variability measures in schizophrenia research (see above, Fernandez et al., 2012), and the purported role of abnormal DA dynamics in this patient group, further motivates the examination of DA-signal variability links in clinical conditions.

Generalizing beyond the brief examples mentioned here, signal variability provides a powerful, theoretically motivated, and biologically plausible measure for understanding any number of individual difference variables already studied in neuroscience.

\section{Conclusions}

Incorporating work from all levels of neuroscience, our intention in this review was to elucidate why researchers should consider employing brain signal variability measures in neuroimaging research, and to discuss some practical issues going forward. It has been known for decades that the brain is inherently variable (Faisal et al., 2008; Pinneo, 1966; Stein et al., 2005; Traynelis and Jaramillo, 1998), and is a dynamic system fluctuating naturally from momentto-moment based on spontaneous reconfiguration or in response to external stimuli (Deco et al., 2011, 2009; Ghosh et al., 2008; Raichle, 2010; Raichle et al., 2007, 2001). The studies reviewed here provide evidence for the importance and sensitivity of brain variability measures in electrophysiology and neuroimaging research that spans human development and aging, brain injury, disease, and cognitive performance across a host of experimental paradigms. As progress continues in brain signal variability research across cellular, systems, computational, and neuroimaging domains, it remains clear that at the very least, we are moving well beyond the conceptualization that signal variability is merely useless noise. If being variable is indeed the natural state of the brain, why not measure and understand it as such? Variability is a crucial "signal" in its own right, and should be considered a next frontier in human brain mapping.

\section{References}

Achard, S., Bassett, D.S., Meyer-Lindenberg, A., Bullmore, E., 2008. Fractal connectivity of long-memory networks. Physical Review E: Statistical, Nonlinear, and Soft Matter Physics 77, 036104.

Andrews-Hanna, J.R., Snyder, A.Z., Vincent, J.L., Lustig, C., Head, D., Raichle, M.E., Buckner, R.L., 2007. Disruption of large-scale brain systems in advanced aging. Neuron 56, 924-935.

Arduini, A., 1963. The tonic discharge of the retina and its central effects. In: Moruzzi, G., Fessard, A., Jasper, H.H. (Eds.), Progress in Brain Research. Elsevier, New York, pp. 184-206.

Arieli, A., Sterkin, A., Grinvald, A., Aertsen, A., 1996. Dynamics of ongoing activity: explanation of the large variability in evoked cortical responses. Science 273, 1868-1871.

Bak, P., Tang, C., Wiesenfeld, K., 1987. Self-organized criticality: An explanation of the $1 / \mathrm{f}$ noise. Physical Review Letters 59, 381-384.

Basalyga, G., Salinas, E., 2006. When response variability increases neural network robustness to synaptic noise. Neural Computation 18, 1349-1379.

Bassett, D.S., Meyer-Lindenberg, A., Achard, S., Duke, T., Bullmore, E., 2006. Adaptive reconfiguration of fractal small-world human brain functional networks. Proceedings of the National Academy of Sciences of the United States of America 103, 19518-19523.

Bäckman, L., Lindenberger, U., Li, S.-C., Nyberg, L., 2010. Linking cognitive aging to alterations in dopamine neurotransmitter functioning: recent data and future avenues. Neuroscience \& Biobehavioral Reviews 34, 670-677.

Bäckman, L., Nyberg, L., Lindenberger, U., Li, S.-C., Farde, L., 2006. The correlative triad among aging, dopamine, and cognition: Current status and future prospects. Neuroscience \& Biobehavioral Reviews 30, 791-807.

Bäckman, L., Nyberg, L., Soveri, A., Johansson, J., Andersson, M., Dahlin, E., Neely, A.S., Virta, J., Laine, M., Rinne, J.O., 2011. Effects of working-memory training on striatal dopamine release. Science 333, 718.

Beck, J.M., Ma, W.J., Kiani, R., Hanks, T., Churchland, A.K., Roitman, J., Shadlen, M.N., Latham, P.E., Pouget, A., 2008. Probabilistic population codes for Bayesian decision making. Neuron 60, 1142-1152.

Beggs, J.M., Plenz, D., 2003. Neuronal avalanches in neocortical circuits. Journal of Neuroscience 23, 11167-11177.

Beggs, J.M., Timme, N., 2012. Being critical of criticality in the brain. Frontiers in Physiology, 3.

Bianciardi, M., Fukunaga, M., van Gelderen, P., Horovitz, S.G., de Zwart, J.A., Shmueli, K., Duyn, J.H., 2009. Sources of functional magnetic resonance imaging signal fluctuations in the human brain at rest: a $7 \mathrm{~T}$ study. Magnetic Resonance Imaging 27, 1019-1029.

Bogdan, R., Carré, J.M., Hariri, A.R., 2012. Toward a mechanistic understanding of how variability in neurobiology shapes individual differences in behavior. Current Topics in Behavioral Neurosciences 12, 361-393.

Bosl, W., Tierney, A., Tager-Flusberg, H., Nelson, C., 2011. EEG complexity as a biomarker for autism spectrum disorder risk. BMC Medicine 9, 18.

Brown, T.G., 1914. On the nature of the fundamental activity of the nervous centres; together with an analysis of the conditioning of rhythmic activity in progression, and a theory of the evolution of function in the nervous system. Journal of Physiology 48, 18-46.

Bullmore, E., Sporns, O., 2009. Complex brain networks: graph theoretical analysis of structural and functional systems. Nature Reviews Neuroscience 10, 186-198.

Burioka, N., Cornélissen, G., Maegaki, Y., Halberg, F., Kaplan, D.T., Miyata, M., Fukuoka, Y., Endo, M., Suyama, H., Tomita, Y., Shimizu, E., 2005. Approximate entropy of the electroencephalogram in healthy awake subjects and absence epilepsy patients. Clinical EEG and Neuroscience 36, 188-193.

Catarino, A., Churches, O., Baron-Cohen, S., Andrade, A., Ring, H., 2011. Atypical EEG complexity in autism spectrum conditions: A multiscale entropy analysis. Clinical Neurophysiology 122, 2375-2383. 
Ciuciu, P., Varoquaux, G., Abry, P., Sadaghiani, S., Kleinschmidt, A., 2012. Scale-free and multifractal time dynamics of fMRI signals during rest and task. Frontiers in Physiology 3, 186.

Cools, R., D'Esposito, M., 2011. Inverted-U-shaped dopamine actions on human working memory and cognitive control. Biological Psychiatry 69, e113-e125.

Costa, M., Goldberger, A., Peng, C.K., 2005. Multiscale entropy analysis of biological signals. Physical Reviews E, 71.

Costa, M., Goldberger, A.L., Peng, C.K., 2002. Multiscale entropy analysis of complex physiologic time series. Physical Review Letters 89, 068102.

Cox, C.L., Uddin, L.Q., Di Martino, A., Castellanos, F.X., Milham, M.P., Kelly, C., 2012. The balance between feeling and knowing: affective and cognitive empathy are reflected in the brain's intrinsic functional dynamics. Social Cognitive and Affective Neuroscience 7, 727-737.

Cox, R.W., 1996. AFNI: software for analysis and visualization of functional magnetic resonance neuroimages. Computers and Biomedical Research 29, 162-173.

Cremer, R., Zeef, E.J., 1987. What kind of noise increases with age? Journal of Gerontology 42, 515-518.

Dauwels, J., Vialatte, F., Cichocki, A., 2010. Diagnosis of alzheimers disease from eeg signals: Where are we standing? Current Alzheimer Research 7, 487-505.

Dayan, P., Walton, M.E., 2012. A step-by-step guide to dopamine. Biological Psychiatry $71,842-843$.

Deco, G., Jirsa, V., McIntosh, A., Sporns, O., Kötter, R., 2009. Key role of coupling, delay, and noise in resting brain fluctuations. Proceedings of the National Academy of Sciences of the United States of America 106, 10302-10307.

Deco, G., Jirsa, V.K., 2012. Ongoing cortical activity at rest: criticality, multistability, and ghost attractors. Journal of Neuroscience 32, 3366-3375.

Deco, G., Jirsa, V.K., McIntosh, A.R., 2011. Emerging concepts for the dynamical organization of resting-state activity in the brain. Nature Reviews Neuroscience 12 , 43-56.

Delorme, A., Makeig, S., 2004. EEGLAB: an open source toolbox for analysis of single-trial EEG dynamics including independent component analysis. Journal of Neuroscience Methods 134, 9-21.

Doya, K., 2008. Modulators of decision making. Nature Neuroscience 11, 410-416.

Escudero, J., Abasolo, D., Hornero, R., Espino, P., López, M., 2006. Analysis of electroencephalograms in Alzheimer's disease patients with multiscale entropy. Physiological Measurement 27, 1091-1106.

Fair, D.A., Cohen, A.L., Power, J.D., Dosenbach, N.U.F., Church, J.A., Miezin, F.M., Schlaggar, B.L., Petersen, S.E., 2009. Functional brain networks develop from a "local to distributed" organization. PLoS Computational Biology 5, e1000381.

Faisal, A.A., Selen, L.P.J., Wolpert, D.M., 2008. Noise in the nervous system. Nature Reviews Neuroscience 9, 292-303.

Fernandez, A., Gomez, C., Hornero, R., López-Ibor, J.J., 2012. Complexity and schizophrenia. Progress in Neuro-Psychopharmacology and Biological Psychiatry, http://dx.doi.org/10.1016/j.pnpbp.2012.03.015.

Fox, M.D.,Snyder, A.Z.,Zacks, J.M., Raichle, M.E., 2005. Nature Neuroscience 9, 23-25

Fraiman, D., Chialvo, D.R., 2012. What kind of noise is brain noise: anomalous scaling behavior of the resting brain activity fluctuations. Frontiers in Physiology, 3.

Friston, K., 2010. The free-energy principle: a unified brain theory? Nature Reviews Neuroscience 11, 127-138.

Friston, K., Ao, P., 2012. Free energy, value, and attractors. Computational and Mathematical Methods in Medicine 2012, 937860.

Friston, K., Breakspear, M., Deco, G., 2012. Perception and self-organized instability. Frontiers in Computational Neuroscience, 6.

Garrett, D.D., Kovacevic, N., McIntosh, A.R., Grady, C.L., 2010. Blood oxygen leveldependent signal variability is more than just noise. Journal of Neuroscience 30 , 4914-4921.

Garrett, D.D., McIntosh, A.R., Grady, C.L., 2011a. Moment-to-moment signal variability in the human brain can inform models of stochastic facilitation now. Nature Reviews Neuroscience 12.

Garrett, D.D., Kovacevic, N., McIntosh, A.R., Grady, C.L., 2011b. The importance of being variable. Journal of Neuroscience 31, 4496-4503.

Garrett, D.D., Kovacevic, N., McIntosh, A.R., Grady, C.L., 2013. The modulation of BOLD variability between cognitive states varies by age and processing speed. Cerebral Cortex 23, 684-693.

Ghosh, A., Rho, Y., McIntosh, A.R., Kötter, R., Jirsa, V.K., 2008. Noise during rest enables the exploration of the brain's dynamic repertoire. PLoS Computational Biology 4, e1000196

Grady, C., 2012. The cognitive neuroscience of ageing. Nature Reviews Neuroscience $13,491-505$

Grady, C.L., Protzner, A.B., Kovacevic, N., Strother, S.C., Afshin-Pour, B., Wojtowicz, M., Anderson, J.A.E., Churchill, N., McIntosh, A.R., 2010. A multivariate analysis of age-related differences in default mode and task-positive networks across multiple cognitive domains. Cerebral Cortex 20, 1432-1447.

Grassberger, P., Procaccia, I., 1983. Characterization of strange attractors. Physical Review Letters 50, 346-349.

Guo, W.-B., Liu, F., Xue, Z.-M., Xu, X.-J., Wu, R.-R., Ma, C.-Q., Wooderson, S.C., Tan, C.-L., Sun, X.-L., Chen, J.-D., Liu, Z.-N., Xiao, C.-Q., Chen, H.-F., Zhao, J.-P., 2012a. Alterations of the amplitude of low-frequency fluctuations in treatment-resistant and treatment-response depression: a resting-state fMRI study. Progress in NeuroPsychopharmacology and Biological Psychiatry 37, 153-160.

Guo, W.-B., Liu, F., Xun, G.-L., Hu, M.-R., Guo, X.-F., Xiao, C.-Q., Chen, H.-F., Wooderson, S.C., Chen, J.-D., Zhao, J.-P., 2012b. Reversal alterations of amplitude of low-frequency fluctuations in early and late onset, first-episode, drug-naive depression. Progress in Neuro-Psychopharmacology and Biological Psychiatry 40C, 153-159.
Hagger-Johnson, G.E., Shickle, D.A., Roberts, B.A., Deary, I.J., 2012. Neuroticism combined with slower and more variable reaction time: synergistic risk factors for 7-year cognitive decline in females. Journals of Gerontology Series B: Psychological Sciences and Social Sciences 67, 572-581.

Han, Y., Wang, J., Zhao, Z., Min, B., Lu, J., Li, K., He, Y., Jia, J., 2011. Frequency-dependent changes in the amplitude of low-frequency fluctuations in amnestic mild cognitive impairment: a resting-state fMRI study. NeuroImage 55, 287-295.

Hariri, A.R., 2009. The neurobiology of individual differences in complex behavioral traits. Annual Review of Neuroscience 32, 225-247.

Hasan, K.M., Sankar, A., Halphen, C., Kramer, L.A., Brandt, M.E., Juranek, J., Cirino, P.T., Fletcher, J.M., Papanicolaou, A.C., Ewing-Cobbs, L., 2007. Development and organization of the human brain tissue compartments across the lifespan using diffusion tensor imaging. Neuroreport 18, 1735-1739.

He, B.J., 2011. Scale-free properties of the functional magnetic resonance imaging signal during rest and task. Journal of Neuroscience 31, 13786-13795.

Heisz, J.J., Shedden, J.M., McIntosh, A.R., 2012. Relating brain signal variability to knowledge representation. NeuroImage 63, 1384-1392.

Hirsh, J.B., Mar, R.A., Peterson, J.B., 2012. Psychological entropy: a framework for understanding uncertainty-related anxiety. Psychological Review 119, 304-320.

Honey, C.J., Kotter, R., Breakspear, M., Sporns, O., 2007. Network structure of cerebra cortex shapes functional connectivity on multiple time scales. Proceedings of the National Academy of Sciences of the United States of America 104, 10240-10245.

Honey, C.J., Sporns, O., Cammoun, L., Gigandet, X., Thiran, J.P., Meuli, R., Hagmann, P., 2009. Predicting human resting-state functional connectivity from structural connectivity. Proceedings of the National Academy of Sciences of the United States of America 106, 2035-2040.

Hoptman, M.J., Zuo, X.-N., Butler, P.D., Javitt, D.C., D’Angelo, D., Mauro, C.J., Milham, M.P., 2010. Amplitude of low-frequency oscillations in schizophrenia: a resting state fMRI study. Schizophrenia Research 117, 13-20.

Jirsa, V.K., Friedrich, R., Haken, H., Kelso, J.A., 1994. A theoretical model of phase transitions in the human brain. Biological Cybernetics 71, 27-35.

Johnson, M.H., 2001. Functional brain development in humans. Nature Reviews Neuroscience 2, 475-483.

Knill, D.C., Pouget, A., 2004. The Bayesian brain: the role of uncertainty in neura coding and computation. Trends in Neurosciences 27, 712-719.

Kruger, G., Glover, G.H., 2001. Physiological noise in oxygenation-sensitive magnetic resonance imaging. Magnetic Resonance in Medicine 46, 631-637.

Kunisato, Y., Okamoto, Y., Okada, G., Aoyama, S., Demoto, Y., Munakata, A., Nomura M., Onoda, K., Yamawaki, S., 2011a. Modulation of default-mode network activity by acute tryptophan depletion is associated with mood change: a resting state functional magnetic resonance imaging study. Neuroscience Research 69 , 129-134.

Kunisato, Y., Okamoto, Y., Okada, G., Aoyama, S., Nishiyama, Y., Onoda, K., Yamawaki, S., 2011b. Personality traits and the amplitude of spontaneous low-frequency oscillations during resting state. Neuroscience Letters 492, 109-113.

Lashley, K.S., 1951. The problem of serial order in behavior. In: Jeffress, L.A. (Ed.) Cerebral Mechanisms in Behavior. Wiley, New York, pp. 112-147.

Laskaris, N.A., Liu, L.C., Ioannides, A.A., 2003. Single-trial variability in early visua neuromagnetic responses: an explorative study based on the regional activation contributing to the N70m peak. NeuroImage 20,765-783.

Leo, A., Bernardi, G., Handjaras, G., Bonino, D., Ricciardi, E., Pietrini, P., 2012. Increased BOLD variability in the parietal cortex and enhanced parieto-occipital connectivity during tactile perception in congenitally blind individuals. Neural Plasticity $2012,720278$.

Li, S.-C., 2012. Neuromodulation of behavioral and cognitive development across the life span. Developmental Psychology 48, 810-814

Li, S.-C., Lindenberger, U., Bäckman, L., 2010. Dopaminergic modulation of cognition across the life span. Neuroscience \& Biobehavioral Reviews 34, 625-630.

Li, S.-C., Lindenberger, U., Sikström, S., 2001. Aging cognition: from neuromodulation to representation. Trends in Cognitive Sciences 5, 479-486.

Li, S.-C., Oertzen, von, T., Lindenberger, U., 2006. A neurocomputational model of stochastic resonance and aging. Neurocomputing 69, 1553-1560.

Lippe, S., Kovacevic, N., McIntosh, A.R., 2009. Differential maturation of brain signal complexity in the human auditory and visual system. Frontiers in Human Neuroscience 3, 48

Lugo, E., Doti, R., Faubert, J., 2008. Ubiquitous crossmodal stochastic resonance in humans: auditory noise facilitates tactile, visual and proprioceptive sensations. PLoS ONE 3, e2860.

Ma, W.J., Beck, J.M., Latham, P.E., Pouget, A., 2006. Bayesian inference with probabilistic population codes. Nature Neuroscience 9, 1432-1438.

Macdonald, S.W.S., Li, S.-C., Bäckman, L., 2009. Neural underpinnings of withinperson variability in cognitive functioning. Psychology and Aging 24 792-808.

Macdonald, S.W.S, Nyberg, L., Bäckman, L., 2006. Intra-individual variability in behavior: links to brain structure, neurotransmission and neuronal activity. Trends in Neurosciences 29, 474-480.

Mattay, V.S., Goldberg, T.E., Fera, F., Hariri, A.R., Tessitore, A., Egan, M.F., Kolachana, B., Callicott, J.H., Weinberger, D.R., 2003. Catechol O-methyltransferase val158met genotype and individual variation in the brain response to amphetamine. Proceedings of the National Academy of Sciences of the United States of America 100, 6186-6191.

McDonnell, M.D., Abbott, D., 2009. What is stochastic resonance? Definitions, misconceptions, debates, and its relevance to biology. PLoS Computational Biology 5, e1000348.

McDonnell, M.D., Ward, L.M., 2011. The benefits of noise in neural systems: bridging theory and experiment. Nature Reviews Neuroscience 12, 415-426. 
McIntosh, A.R., Kovacevic, N., Itier, R.J., 2008. Increased brain signal variability accompanies lower behavioral variability in development. PLoS Computational Biology 4, e1000106.

McIntosh, A.R., Kovacevic, N., Lippe, S., Garrett, D., Grady, C., Jirsa, V., 2010. The development of a noisy brain. Archives Italiennes de Biologie 148, 323-337.

Mennes, M., Zuo, X.-N., Kelly, C., Di Martino, A., Zang, Y.-F., Biswal, B., Castellanos, F.X., Milham, M.P., 2011. Linking inter-individual differences in neural activation and behavior to intrinsic brain dynamics. Neurolmage 54 , 2950-2959.

Mišić, B., Mills, T., Taylor, M.J., McIntosh, A.R., 2010. Brain noise is task dependent and region specific. Journal of Neurophysiology 104, 2667-2676.

Mišić, B., Vakorin, V.A., Paus, T., McIntosh, A.R., 2011a. Functional embedding predicts the variability of neural activity. Frontiers in Systems Neuroscience 5, 90.

Mišić, B., Vakorin, V.A., Kovacevic, N., Paus, T., McIntosh, A.R., 2011b. Extracting message inter-departure time distributions from the human electroencephalogram. PLoS Computational Biology 7, e1002065.

Mitra, P.P., Bokil, H., 2008. Observed Brain Dynamics. Oxford University Press, New York, NY.

Mizuno, T., Takahashi, T., Cho, R.Y., Kikuchi, M., Murata, T., Takahashi, K., Wada, Y., 2010. Assessment of EEG dynamical complexity in Alzheimer's disease using multiscale entropy. Clinical Neurophysiology 121, 1438-1446.

Montague, P.R., Hyman, S.E., Cohen, J.D., 2004. Computational roles for dopamine in behavioural control. Nature 431, 760-767.

Nenadovic, V., Hutchison, J.S., Dominguez, L.G., Otsubo, H., Gray, M.P., Sharma, R., Belkas, J., Velazquez, J.L.P., 2008. Fluctuations in Cortical Synchronization in Pediatric Traumatic Brain Injury. Journal of Neurotrauma 25, 615-627.

Neumann, von, J., Kent, R.H., Bellinson, H.R., 1941. The mean square successive difference. The Annals of Mathematical Statistics 12, 153-162.

Nir, Y., Mukamel, R., Dinstein, I., Privman, E., Harel, M., Fisch, L., Gelbard-Sagiv, H., Kipervasser, S., Andelman, F., Neufeld, M.Y., Kramer, U., Arieli, A., Fried, I., Malach, R., 2008. Interhemispheric correlations of slow spontaneous neuronal fluctuations revealed in human sensory cortex. Nature Neuroscience 11, 1100-1108.

Niv, Y., Daw, N.D., Joel, D., Dayan, P., 2007. Tonic dopamine: opportunity costs and the control of response vigor. Psychopharmacology (Berlin) 191, 507-520.

Niv, Y., Schoenbaum, G., 2008. Dialogues on prediction errors. Trends in Cognitive Sciences 12, 265-272.

Park, J.-H., Kim, S., Cheol-Hyun, K., Cichocki, A., Kim, K., 2007. Multiscale entropy analysis of EEG from patients under different pathological conditions. Fractals $15,399-404$.

Pincus, S.M., 1991. Approximate entropy as a measure of system complexity. Proceedings of the National Academy of Sciences of the United States of America $88,2297-2301$.

Pinneo, L.R., 1966. On noise in the nervous system. Psychological Review 73, $242-247$.

Plenz, D., Thiagarajan, T.C., 2007. The organizing principles of neuronal avalanches: cell assemblies in the cortex? Trends in Neurosciences 30, 101-110.

Power, J.D., Fair, D.A., Schlaggar, B.L., Petersen, S.E., 2010. The development of human functional brain networks. Neuron 67, 735-748.

Protzner, A.B., Valiante, T., Kovacevic, N., McCormick, C., McAndrews, M.P., 2010. Hippocampal signal complexity in mesial temporal lobe epilepsy: a noisy brain is a healthy brain. Archives Italiennes de Biologie 148, 289-297.

Raichle, M.E., 2010. Two views of brain function. Trends in Cognitive Sciences 14, 180-190.

Raichle, M.E., MacLeod, A.M., Snyder, A.Z., Powers, W.J., Gusnard, D.A., Shulman, G.L., 2001. A default mode of brain function. Proceedings of the National Academy of Sciences of the United States of America 98, 676-682.

Raichle, M.E., Snyder, A.Z., Raichle, M.E., Snyder, A.Z., 2007. A default mode of brain function: a brief history of an evolving idea. Neurolmage 37, 1097-1099, 108390- discussion.

Raja Beharelle, A., Kovacevic, N., McIntosh, A.R., Levine, B., 2012. Brain signal variability relates to stability of behavior after recovery from diffuse brain injury. Neurolmage 60, 1528-1537.

Raz, N., 2005. Regional brain changes in aging healthy adults: general trends, individual differences and modifiers. Cerebral Cortex 15, 1676-1689.

Raz, N., Rodrigue, K.M., 2006. Differential aging of the brain: Patterns, cognitive correlates and modifiers. Neuroscience \& Biobehavioral Reviews 30, 730-748.

Redcay, E., Kennedy, D.P., Courchesne, E., 2007. fMRI during natural sleep as a method to study brain function during early childhood. NeuroImage 38, 696-707.

Richman, J.S., Moorman, J.R., 2000. Physiological time-series analysis using approximate entropy and sample entropy. American Journal of Physiology - Heart and Circulatory Physiology 278, H2039-H2049.

Robinson, M.D., Tamir, M., 2005. Neuroticism as mental noise: a relation between neuroticism and reaction time standard deviations. Journal of Personality and Social Psychology 89, 107-114.

Salthouse, T.A., Lichty, W., 1985. Tests of the neural noise hypothesis of age-related cognitive change. Journal of Gerontology 40, 443-450.

Samanez-Larkin, G.R., Kuhnen, C.M., Yoo, D.J., Knutson, B., 2010. Variability in nucleus accumbens activity mediates age-related suboptimal financial risk taking. Journal of Neuroscience 30, 1426-1434.

Schomer, D.L., Lopes da Silva, F.H. (Eds.), 2011. Niedermeyer's Electroencephalography: Basic Principles, Clinical Applications, and Related Fields. , 6th ed. Lippincott Williams \& Wilkins, Philadelphia, PA.
Shannon, C.E., 1948. A mathematical theory of communication. The Bell System Technical Journal 27, 379-423, 623-656.

Shew, W.L., Plenz, D., 2013. The functional benefits of criticality in the cortex. Neuroscientist $19,88-100$.

Shew, W.L., Yang, H., Petermann, T., Roy, R., Plenz, D., 2009. Neuronal avalanches imply maximum dynamic range in cortical networks at criticality. Journal of Neuroscience 29, 15595-15600.

Shew, W.L., Yang, H., Yu, S., Roy, R., Plenz, D., 2011. Information capacity and transmission are maximized in balanced cortical networks with neuronal avalanches. Journal of Neuroscience 31, 55-63.

Song, X.-W., Dong, Z.-Y., Long, X.-Y., Li, S.-F., Zuo, X.-N., Zhu, C.-Z., He, Y., Yan, C.-G., Zang, Y.-F., 2011. REST: a toolkit for resting-state functional magnetic resonance imaging data processing. PLoS ONE 6, e25031.

Sowell, E.R., Peterson, B.S., Thompson, P.M., Welcome, S.E., Henkenius, A.L., Toga, A.W., 2003. Mapping cortical change across the human life span. Nature Neuroscience 6, 309-315.

Sowell, E.R., Thompson, P.M., Leonard, C.M., Welcome, S.E., Kan, E., Toga, A.W., 2004. Longitudinal mapping of cortical thickness and brain growth in normal children. Journal of Neuroscience 24, 8223-8231.

Stam, C.J., 2005. Nonlinear dynamical analysis of EEG and MEG: Review of an emerging field. Clinical Neurophysiology 116, 2266-2301.

Stein, R.B., Gossen, E.R., Jones, K.E., 2005. Neuronal variability: noise or part of the signal? Nature Reviews Neuroscience 6, 389-397.

Suckling, J., Wink, A.M., Bernard, F.A., Barnes, A., Bullmore, E., 2008. ScienceDirect.com - Journal of Neuroscience Methods - Endogenous multifractal brain dynamics are modulated by age, cholinergic blockade and cognitive performance. Journal of Neuroscience Methods 174, 292-300.

Supekar, K., Musen, M., Menon, V., 2009. Development of large-scale functional brain networks in children. PLoS Biology 7, e1000157.

Tagliazucchi, E., Balenzuela, P., Fraiman, D., Chialvo, D.R., 2012. Criticality in largescale brain FMRI dynamics unveiled by a novel point process analysis. Frontiers in Physiology 3, 15.

Takahashi, T., 2012. Complexity of spontaneous brain activity in mental disorders. Progress in Neuro-Psychopharmacology and Biological Psychiatry, http://dx.doi.org/10.1016/j.pnpbp.2012.05.001.

Takahashi, T., Cho, R.Y., Mizuno, T., Kikuchi, M., Murata, T., Takahashi, K., Wada, Y., 2010. Antipsychotics reverse abnormal EEG complexity in drug-naive schizophrenia: A multiscale entropy analysis. NeuroImage 51, $173-182$.

Takahashi, T., Cho, R.Y., Murata, T., Mizuno, T., Kikuchi, M., Mizukami, K., Kosaka, H., Takahashi, K., Wada, Y., 2009. Age-related variation in EEG complexity to photic stimulation: A multiscale entropy analysis. Clinical Neurophysiology 120 , 476-483.

Toga, A.W., Thompson, P.M., Sowell, E.R., 2006. Mapping brain maturation. Trends in Neurosciences 29, 148-159.

Tononi, G., 1998. Complexity and coherency: integrating information in the brain. Trends in Cognitive Sciences 2, 474-484.

Tononi, G., Sporns, O., Edelman, G.M., 1994. A measure for brain complexity: relating functional segregation and integration in the nervous system. Proceedings of the National Academy of Sciences of the United States of America 91, 5033-5037.

Traynelis, S.F., Jaramillo, F., 1998. Getting the most out of noise in the central nervous system. Trends in Neurosciences 21, 137-145.

Uddin, L.Q., Supekar, K.S., Ryali, S., Menon, V., 2011. Dynamic reconfiguration of structural and functional connectivity across core neurocognitive brain networks with development. Journal of Neuroscience 31, 18578-18589.

Vakorin, V.A., Lippe, S., McIntosh, A.R., 2011. Variability of brain signals processed locally transforms into higher connectivity with brain development. Journal of Neuroscience 31, 6405-6413.

van Leeuwen, C., 2008. Chaos breeds autonomy: connectionist design between bias and baby-sitting. Cognitive Processing 9, 83-92.

Varela, F., Lachaux, J.P., Rodriguez, E., Martinerie, J., 2001. The brainweb: phase synchronization and large-scale integration. Nature Reviews Neuroscience 2, 229-239.

Ward, L.M., 2003. Synchronous neural oscillations and cognitive processes. Trends in Cognitive Sciences 7, 553-559.

Ward, L.M., Doesburg, S.M., Kitajo, K., MacLean, S.E., Roggeveen, A.B., 2006. Neural synchrony in stochastic resonance, attention, and consciousness. Canadian Journal of Experimental Psychology/Revue canadienne de psychologie expérimentale 60, 319-326.

Welford, A.T., 1965. Performance, biological mechanisms and age: A theoretical sketch. In: Welford, A.T., Birren, J.E. (Eds.), Behavior, Aging, and the Nervous System. Charlse C. Thomas, Springfield, IL.

Welford, A.T., 1981. Signal, noise, performance, and age. Human Factors 23, 97-109.

Westlye, L.T., Walhovd, K.B., Dale, A.M., Bjornerud, A., Due-Tonnessen, P., Engvig, A., Grydeland, H., Tamnes, C.K., Ostby, Y., Fjell, A.M., 2010. Life-span changes of the human brain white matter: diffusion tensor imaging (DTI) and volumetry. Cerebral Cortex 20, 2055-2068.

Wutte, M.G., Smith, M.T., Flanagin, V.L., Wolbers, T., 2011. Physiological signal variability in hMT+ reflects performance on a direction discrimination task. Frontiers in Psychology 2, 185.

Xi, Q., Zhao, X., Wang, P., Guo, Q., Jiang, H., Cao, X., He, Y., Yan, C., 2012. Spontaneous brain activity in mild cognitive impairment revealed by amplitude of lowfrequency fluctuation analysis: a resting-state fMRI study. Radiology Medicine $117,865-871$. 
Yang, H., Shew, W.L., Roy, R., Plenz, D., 2012. Maximal variability of phase synchrony in cortical networks with neuronal avalanches. Journal of Neuroscience 32, 1061-1072.

Yu-Feng, Z., Yong, H., Chao-Zhe, Z., Oing-Jiu, C., Man-Oiu, S., Meng, L., Li-Xia, T., TianZi, J., Yu-Feng, W., 2007. Altered baseline brain activity in children with ADHD revealed by resting-state functional MRI. Brain and Development 29, 83-91.

Zalesky, A., Fornito, A., Bullmore, E., 2012. On the use of correlation as a measure of network connectivity. NeuroImage 60, 2096-2106.

Zandi, A.S., Dumont, G.A., Javidan, M., Tafreshi, R., 2009. An entropy-based approach to predict seizures in temporal lobe epilepsy using scalp EEG. Conference Proceedings of the IEEE Engineering in Medicine and Biology Society 2009, $228-231$.
Zhu, Z., Lu, O., Meng, X. Jiang, Q. Peng, L., Wang, Q, 2012. Spatial patterns of intrinsic neural activity in depressed patients with vascular risk factors as revealed by the amplitude of low-frequency fluctuation. Brain Research 1483 82-88.

Zou, Q.-H., Zhu, C.-Z., Yang, Y., Zuo, X.-N., Long, X.-Y., Cao, Q.-J., Wang, Y.-F., Zang, Y.-F., 2008. An improved approach to detection of amplitude of low-frequency fluctuation (ALFF) for resting-state fMRI: Fractional ALFF. Journal of Neuroscience Methods 172, 137-141.

Zuo, X.-N., Di Martino, A., Kelly, C., Shehzad, Z.E., Gee, D.G., Klein, D.F., Castellanos, F.X., Biswal, B.B., Milham, M.P., 2010. The oscillating brain: Complex and reliable. NeuroImage 49, 1432-1445. 